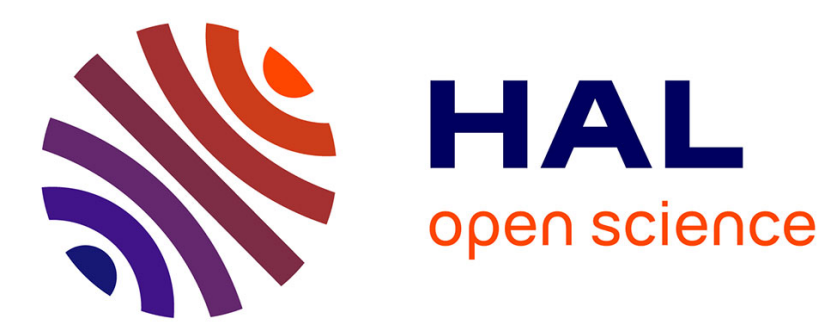

\title{
A micromechanical model of a hard interface with micro-cracking damage
}

Maria Letizia Raffa, Frédéric Lebon, Raffaella Rizzoni

\section{To cite this version:}

Maria Letizia Raffa, Frédéric Lebon, Raffaella Rizzoni. A micromechanical model of a hard interface with micro-cracking damage. International Journal of Mechanical Sciences, 2022, 216, pp.106974. 10.1016/j.ijmecsci.2021.106974 . hal-03404654

\section{HAL Id: hal-03404654 \\ https://hal.science/hal-03404654}

Submitted on 26 Oct 2021

HAL is a multi-disciplinary open access archive for the deposit and dissemination of scientific research documents, whether they are published or not. The documents may come from teaching and research institutions in France or abroad, or from public or private research centers.
L'archive ouverte pluridisciplinaire $\mathbf{H A L}$, est destinée au dépôt et à la diffusion de documents scientifiques de niveau recherche, publiés ou non, émanant des établissements d'enseignement et de recherche français ou étrangers, des laboratoires publics ou privés. 


\title{
A micromechanical model of a hard interface with micro-cracking damage
}

\author{
Maria Letizia Raffa ${ }^{\mathrm{a}, *}$, Frédéric Lebon ${ }^{\mathrm{b}}$, Raffaella Rizzcı. \\ ${ }^{a}$ Laboratoire QUARTZ, EA 7393, ISAE-Supméca, 3 rue Fernand H inaน:9 9407 \\ Saint-Ouen, France \\ ${ }^{b}$ Aix Marseille Univ, CNRS, Centrale Marseille, LMA, 4 impasse viko. : Tt , la 13453 \\ Marseille Cedex 13, France \\ ${ }^{c}$ Department of Engineering, University of Ferrara, via Saragat ' 44122 Ferrara, Italy
}

\section{Abstract}

Bonding techniques are increasingly used . $\mathrm{n}_{\mathrm{c}} \mathrm{vv}$ industrial fields. Modelling the under-load damaging behavior o: araru structural adhesives is still an open challenge. This work proposes a $\mathrm{n} 、$ v hard interface analytical model with evolutive micro-cracking damage. The nodel is obtained within a rigorous theoretical framework combining c 'vn ptotic theory and micromechanical homogenization. Main new feat" ies are: (i) the adoption of two dual homogenization approaches; (ii) $t^{t} 、$ for $\_$ulation of a thermodynamically-based damage evolution law for : ard . terfaces. The interface model is able to describe both ductile and bri le c..mage behavior of hard structural adhesives. Provided examples on ths s. uctural behavior, under several loads, suggest the suitability of the $\rho_{1}$ n'ssed interface model as a modelling strategy for hard structural adhe ; ve; with micro-cracking damage.

\footnotetext{
*Corresponr'ng uthor

Email addre s: maria-letizia.raffa@isae-supmeca.fr (Maria Letizia Raffa), lebon@lma.cnrs-mi .fr (Frédéric Lebon), raffaella.rizzoni@unife.it (Raffaella Rizzoni)
} 
Keywords: bonding, adhesives, damage, imperfect interfaces, homogenization, asymptotic theory

2

\section{Introduction}

Bonding has become a very common practice to assembly $\mathrm{m}_{\iota}$ rir is and structural elements in many industrial fields, such as aeron zutı, spatial, automotive, nuclear, civil, mechanical and bio-engineering majnly because structural adhesives offer low-cost techniques and a great design freedom

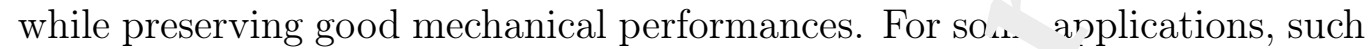
as assemblies of fiber-reinforced composites and impl int 1 xations, bonding is the only viable assembly technology. To achievn be s " nerformances avoiding too large mismatch in terms of thermo-elastic ${ }_{2}{ }^{\prime} \jmath_{\text {Jpe }}$. ies, structural adhesives and adherents have, in some cases, an equir 'ent stiffness. Some examples can be cited: acrylic adhesives, whose Youı ' modulus $(E)$ is around 2-3 GPa [1, are used in manufacture of plyv s' ( $E=5-8 \mathrm{GPa})$; phenolic and epoxy adhesives with $E=3-5 \mathrm{GPa}[\mathrm{O}]$ are used to bond structures of GFRP (polyester-glass composites) with $\Gamma=\perp 5-28 \mathrm{GPa}$; orthodontic adhesives with $E=18-22 \mathrm{GPa}[3]$ ar us ly used for cementation of brackets on enamel $(E \simeq 65 \mathrm{GPa})$.

An adhesive equally stir ${ }_{\wedge r} \mathrm{t}$ tan adherents is defined, from a mechanical point of view, as a $h_{1}$ ' i..terface, as opposed to the definition of soft interface [4, 5]. A wide 'ite ature exists concerning models of soft material interfaces, including interface model $, \mathrm{ft}^{\prime}, \mathrm{n}$ take into account the nonlinear evolution of the interface properties by introducing at least one parameter (of damage, adhesion, 
etc.) whose variation depends macroscopically on kinematic variables [6 15]. Numerical soft interface models, in the framework of the finite element theory, generally use cohesive zone models (CZM) based on traction-sepr ration laws of various shapes, to describe cohesive and adhesive failure [16 $\angle 4]$.

Recently, some analytical models of hard material interfaces hav been also developed [23 29] and it has been proved that interface mo iels 'eve oped for soft adhesives cannot be directly applied in the case of har aunesives [25]. Moreover, the existing hard interface models do not consider w. degradation of the adhesive material properties.

This paper provides a novelty within this conter $\mathrm{t}, \mathrm{x}^{\mathrm{t}}$ proposing a hard material interface model accounting for an evoluti - mıcro-cracking damage. In the last twenty years, the present authors estabı. 'cd an original modelling strategy to derive soft and hard imperfect inte_-ice models based on the combination of asymptotic theory and mics, ner hanical homogenization [11, [14, 23 26] (see Fig. 1). This strategy nes iready been successfully used to describe the mechanics of thin elastic layers in adhesive-like problems and contact problems [13, 15, 30, 31]. 'oreover, it has been identified as a sound alternative to the classica' nhesive zone models, principally because imperfect interface models all $r$. consider the physics of the adhesives in terms of geometrical (thickness, urface roughness), mechanical (anisotropy, non-linearity) and damag, nro erties.

This work is an ext n $n$ io 1 of the authors' modelling strategy of hard imperfect interfaces. D wing on Kachanov's micromechanical homogenization theory [32 37], nicr s-cracking damage is represented by a microcracks density parameter. Partı ularly, the adoption of a generalized cracks density [38] 


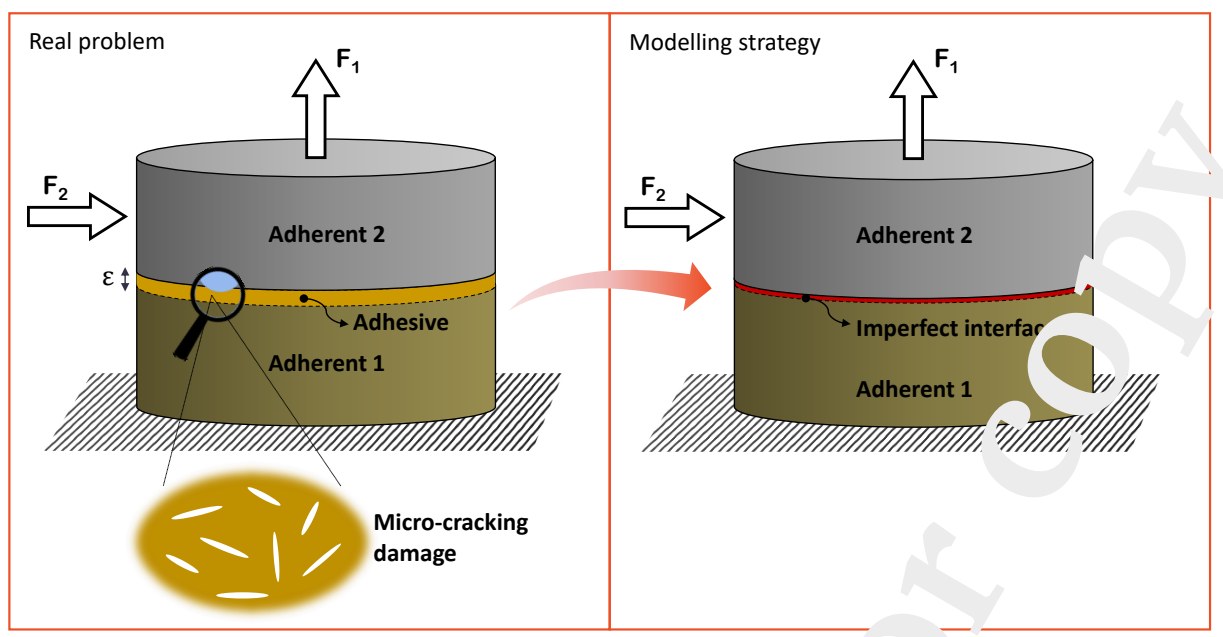

Fig. 1: Schematic sketch of the imperfect inヶ ace ı.ıdelling strategy

allows to by-pass the geometrical definitio . of the cracks, which is possible only for circular and regular cracks [32], ; nrı c a matter of fact it extends the generality of the proposed interface model to any regular and irregular cracks shape. It should also be noted that the _eneralized microcracks density can be measured postmortem by $\mathrm{X}-1$ micro-tomography [15]. The evolutive character of the micro-crackir ? a - age is described by introducing a new evolution law of the generalizeu acks density.

The paper is structur, ' as follows. The hard imperfect interface law is derived via the asymp ot of $^{1}$ expansions method in Section 2. In Section 3, the microcracked-me ${ }^{2}$ rial-interface properties are derived through two dual approaches of $r$ icr mechanical homogenization, stress [32, 33] and strainbased [39, 40]. The c amage evolution law is derived from a thermodynamic 
approach and then included in the hard interface model via the asymptotic expansions method. In Section 4, the behavior of the proposed interface model under various loading type is discussed via some academic exa nples. Moreover, the influence of damage parameters is investigated. Coilciums ns and perspectives are drawn at the end of the paper.

\section{Derivation of the hard imperfect interface model}

\subsection{Notation and problem statement}

The herein adopted matched asymptotic expansion $\imath_{\text {ـ. }}$-ry builds on the tradition of using asymptotic analysis to derive me han cal laws governing imperfect interface conditions [41-48].

In what follows, a thin material layer of $c$ - nstar.c thickness $t$ embedded between at least two solids is referred as intc. hase. Being $L$ a representative length scale of the geometry, the non-diı nsional interphase thickness $\varepsilon=t / L$ can be defined and taken as a $s^{2}{ }^{1} /$ parameter for the asymptotic expansions of the elastic problem. " ' hen $\varepsilon \ll 1$, the thin layer can be substituted by a surface separating $\mathrm{tt}$. - dherents called interface across which

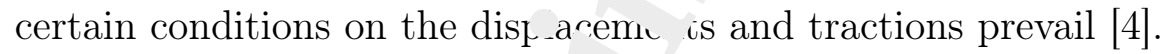

The interphase occupies n domain $\mathcal{B}^{\varepsilon}$ with cross-section $\mathcal{S}, \mathcal{S}$ being an open bounded set in $\mathbb{R}^{2}$ wit $^{+}$. snooth boundary. The adherents occupy the reference configurations ()$^{\varepsilon}{ }_{ \pm} \cdots \mathbb{R}^{3}$. Let $\mathcal{S}^{\varepsilon}{ }_{ \pm}$be taken to denote the plane interfaces between inte ${ }_{\perp_{1}}{ }^{h}{ }$ se and adherents and let $\Omega^{\varepsilon}=\Omega^{\varepsilon}{ }_{ \pm} \cup \mathcal{S}^{\varepsilon}{ }_{ \pm} \cup \mathcal{B}^{\varepsilon}$ denote the whole $\sim^{n} n_{p} \rightarrow i$ se system. It is assumed that the displacement and stress vector ficı. ' re continuous across $\mathcal{S}^{\varepsilon}{ }_{ \pm}$.

An orthonormal Cartesian basis $\left(O, \mathbf{i}_{1}, \mathbf{i}_{2}, \mathbf{i}_{3}\right)$ is introduced and let $\left(x_{1}, x_{2}, x_{3}\right)$ 

belongs to $\mathcal{S}$. The aforementioned system is sketched in Fig. 2 a.

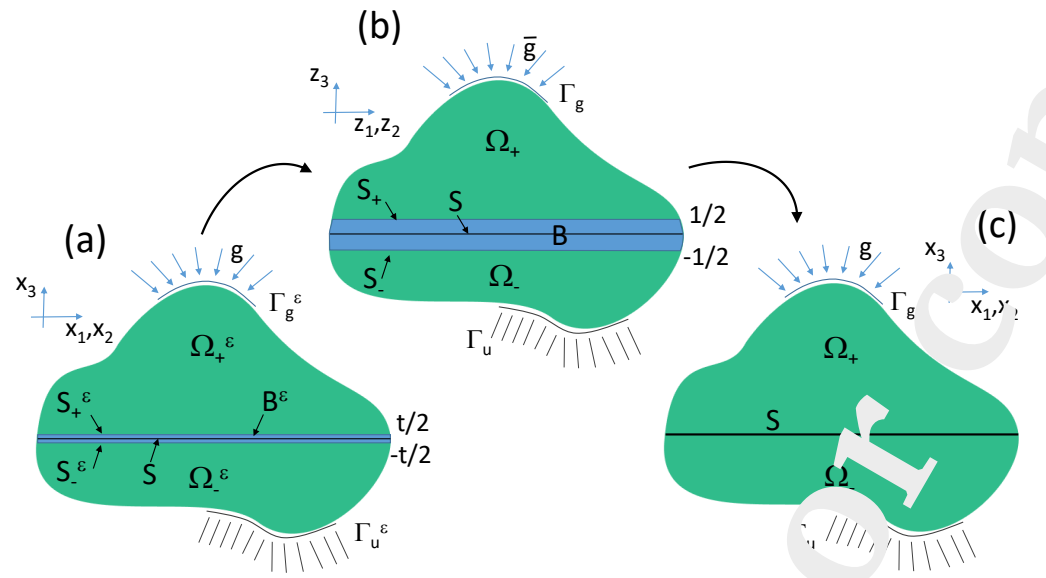

Fig. 2: The three steps of the matched asymptotic $e_{\ldots_{+}}$?nsion method: (a) Reference configuration (interphase); (b) Rescaled confıg ${ }$. ion (asymptotic expansion phase); (c) Limit configuration (interface).

be taken to denote the three coordinates of a particle. The origin of the basis

The materials of the composite s'stem are assumed to be homogeneous and linearly elastic and let $\mathbb{A}_{ \pm}, \mathbb{B}^{\varepsilon}$ he une fourth-rank elasticity tensors of adherents and of interphase, respe +ively. Tensors $\mathbb{A}_{ \pm}, \mathbb{B}^{\varepsilon}$ have the usual symmetry properties, with th mı or and major symmetries, and are positive definite. Note that an' ssı mption on the anisotropy of adhesive and adherents materials is neeal' for the proposed development. As a matter of fact, it extends the ger "al' y of the proposed asymptotic approach to any anisotropic material

Adherents 2. $\quad$ \& ojected to a body force density $\mathbf{f}^{ \pm}: \Omega^{\varepsilon}{ }_{ \pm} \mapsto \mathbb{R}^{3}$ and to a surface force density $\mathbf{g}^{ \pm}: \Gamma_{g}^{\varepsilon} \mapsto \mathbb{R}^{3}$ on $\Gamma_{g}^{\varepsilon} \subset\left(\partial \Omega^{\varepsilon} \backslash \backslash \mathcal{S}_{+}^{\varepsilon}\right) \cup\left(\partial \Omega^{\varepsilon} \backslash \backslash \mathcal{S}_{-}^{\varepsilon}\right)$. Body 
forces in the interphase are neglected.

On $\Gamma_{u}^{\varepsilon}=\left(\partial \Omega^{\varepsilon}+\backslash \mathcal{S}_{+}^{\varepsilon}\right) \cup\left(\partial \Omega_{-}^{\varepsilon} \backslash \mathcal{S}_{-}^{\varepsilon}\right) \backslash \Gamma_{g}^{\varepsilon}$, homogeneous boundary conditions are prescribed:

$$
\mathbf{u}^{\varepsilon}=\mathbf{0} \quad \text { on } \Gamma_{u}^{\varepsilon},
$$

where $\mathbf{u}^{\varepsilon}: \Omega^{\varepsilon} \mapsto \mathbb{R}^{3}$ is the displacement field defined on $\Omega^{\varepsilon}$. ${ }^{\nu} \cdot{ }^{\prime}{ }^{\prime}$ daries $\Gamma_{g}^{\varepsilon}, \Gamma_{u}^{\varepsilon}$ are assumed to be located sufficiently far from the intes rhas s and the external boundaries of the interphase $\mathcal{B}^{\varepsilon}\left(\partial \mathcal{S} \times\left(-\frac{\varepsilon}{2}, \frac{\varepsilon}{2}\right)\right)$ a] a ass umed to be stress-free. The external forces field is endowed with sufficient regularity to ensure the existence of an equilibrium configuration [2.0].

The following notation is adopted:

- $[f]:=f\left(\mathbf{z}_{\alpha}, \frac{1}{2}\right)-f\left(\mathbf{z}_{\alpha},-\frac{1}{2}\right) \Rightarrow$ jump in the re - alea configuration (Fig. $\left.2 \mathrm{~b}\right)$;

- $\langle f\rangle:=\int_{-\frac{1}{2}}^{\frac{1}{2}} f\left(\mathbf{z}_{\alpha}, z_{3}\right) d z_{3} \Rightarrow$ average in tl rescaled configuration;

- $[[f]]:=f\left(\mathbf{x}_{\alpha}, 0^{+}\right)-f\left(\mathbf{x}_{\alpha}, 0^{-}\right) \Rightarrow$ jum $\left._{\boldsymbol{r}}\right\urcorner$ the limit configuration (Fig. 2f);

- $\langle\langle f\rangle\rangle:=\frac{1}{2}\left(f\left(\mathbf{x}_{\alpha}, 0^{+}\right)+f\left(\mathbf{x}_{\alpha}, 0^{-)}\right) \Rightarrow\right.$ average in the limit configuration;

where $f$ is a generic function, $\mathbf{z}_{\alpha}==\left(\sim, z_{2}\right)$ and $\mathbf{x}_{\alpha}=\left(x_{1}, x_{2}\right)$.

\subsection{The one-order asymptoti, the :y}

This section details the in steps of the asymptotic analysis leading to the hard interface law at onc ' der. Full formulation is reported in Appendix $\mathrm{A}$ and more details cou ' $\mathrm{h}$ ' found in [23 26].

Generally, the oldi $i^{\prime}$ y tensor $\mathbb{B}^{\varepsilon}$ of a hard interphase does not depend on $\varepsilon$ [23, 25]:

$$
\mathbb{B}^{\varepsilon}=\mathbb{B}
$$


116 In the rescaled configuration (Fig. 2b) and considering Eqs. A.7) and ${ }_{117}$ A.14b, the stress-strain equation A.31b reads as:

$$
\hat{\boldsymbol{\sigma}}^{0}+\varepsilon \hat{\boldsymbol{\sigma}}^{1}=\mathbb{B}\left(\varepsilon^{-1} \hat{\mathbf{e}}^{-1}+\hat{\mathbf{e}}^{0}+\varepsilon \hat{\mathbf{e}}^{1}\right)+o(\varepsilon)
$$

Equation (3) is true $\forall \varepsilon$, thus the following conditions are derived

$$
\begin{aligned}
\mathbf{0} & =\mathbb{B}\left(\hat{\mathbf{e}}^{-1}\right) \\
\hat{\boldsymbol{\sigma}}^{0} & =\mathbb{B}\left(\hat{\mathbf{e}}^{0}\right)
\end{aligned}
$$

By considering Eq. A.8 and the positive definiteness of $t$ e tensor $\mathbb{B}$, Eq. 4a gives:

$$
\hat{\mathbf{u}}_{, 3}^{0}=0 \Rightarrow\left[\hat{\mathbf{u}}^{0}\right]=\mathbf{0}
$$

Moreover, substituting Eq. (A.9) written for $k={ }^{\prime}$ into Eq. (4b) it gives:

$$
\hat{\boldsymbol{\sigma}}^{0} \mathbf{i}_{j}=\mathbf{K}^{1 j} \hat{\mathbf{u}}_{, 1}^{0}+\mathbf{K}^{2 j} \hat{\mathbf{u}}_{, 2}^{0} \cdot \mathbf{K}^{\lrcorner} \hat{\mathbf{u}}_{, 3}^{1}
$$

with $j=1,2,3$ and $\mathbf{K}^{j l}$ being the two-or $\cdots$ tensors such that $K_{k i}^{j l}:=B_{i j k l}$. Next, integrating Eq. (6) with respect tc $-_{3}$ (for $j=3$ ) and considering Eq. A.17 it results:

$$
\left[\hat{\mathbf{u}}^{1}\right]=\left(\mathbf{K}^{3^{\circ},-1}\left(\mathcal{J}^{0} \mathbf{i}_{3}-\mathbf{K}^{\alpha 3} \hat{\mathbf{u}}_{, \alpha}^{0}\right)\right.
$$

124 Then, by replacing Eq. (6) $(j=1, \dot{2})$ in the equilibrium equation A.18) one obtains:

$$
\left(\hat{\boldsymbol{\sigma}}^{1} \mathbf{i}_{3}\right)_{, 3}=-\left({ }_{\nu} \hat{i}_{\alpha}\right)_{, \alpha}-\left(\mathbf{K}^{1 \alpha} \hat{\mathbf{u}}_{, 1}^{0}+\mathbf{K}^{2 \alpha} \hat{\mathbf{u}}_{, 2}^{0}+\mathbf{K}^{3 \alpha} \hat{\mathbf{u}}_{, 3}^{1}\right)_{, \alpha}
$$

126 Next, by integrating $`$. (8) with respect to $z_{3}$ between $-1 / 2$ and $1 / 2$ and ${ }_{27}$ by using Eq. (7) it s obtained:

$$
\left[\hat{\boldsymbol{\sigma}}^{1} \mathbf{i}_{3}\right]=\left(-\mathbf{K}^{\beta \alpha} \hat{\mathbf{u}}_{, \beta}^{0}-\mathbf{K}^{3 \alpha}\left(\mathbf{K}^{33}\right)^{-1}\left(\hat{\boldsymbol{\sigma}}^{0} \mathbf{i}_{3}-\mathbf{K}^{\beta 3} \hat{\mathbf{u}}_{, \beta}^{0}\right)\right)_{, \alpha}
$$


where Greek indexes $(\alpha, \beta=1,2)$ are related to the in-plane $\left(x_{1}, x_{2}\right)$ quantities. Note that in Eq. (9) higher order effects, related to in-plane derivatives, appear. These terms, usually neglected in standard zero-order $\mathrm{t}^{1}$ eories [23, 25], are related to the curvature of the deformed interface (seccnu-v, ${ }^{\text {ter }}$ derivatives).

Finally, the transition from the rescaled configuration to the $11 \ldots$ t, co .figuration is obtained by introducing the matching conditions $\mathrm{E}_{\mathbf{4}}$. A.27)-A.30 and the interface laws at both zero-order and one-order are uuıved:

- Zero-order interface law:

$$
\begin{aligned}
& {\left[\left[\mathbf{u}^{0}\right]\right]=\mathbf{0}} \\
& {\left[\left[\boldsymbol{\sigma}^{0} \mathbf{i}_{3}\right]\right]=\mathbf{}}
\end{aligned}
$$

- One-order interface law:

$$
\begin{aligned}
{\left[\left[\mathbf{u}^{1}\right]\right]=\left(\mathbf{K}^{33}\right)^{-1}\left(\boldsymbol{\sigma}^{0} \mathbf{i}_{3}-\mathbf{K}^{\alpha 3} \mathbf{u}_{, u}{ }^{\prime}-\left\langle\left\langle\mathbf{u}_{, 3}^{0}\right\rangle\right\rangle\right.} \\
{\left[\left[\boldsymbol{\sigma}^{1} \mathbf{i}_{3}\right]\right]=\left(-\mathbf{K}^{\beta \alpha} \mathbf{u}_{, \beta}^{0}-{ }^{-{ }^{3 \alpha}}\left(\mathbf{K}^{33}\right)^{-1}\left(\boldsymbol{\sigma}^{0} \mathbf{i}_{3}-\mathbf{K}^{\beta 3} \mathbf{u}_{, \beta}^{0}\right)\right)_{, \alpha} } \\
\quad-\left\langle\left\langle\boldsymbol{\sigma}_{3}^{0} \mathbf{i}_{\text {is/, }}\right.\right.
\end{aligned}
$$

Equations (10)-(11) are the su rard perfect interface condition, characterized by the continuity in $t^{r}$ ms of displacements and stresses at the interface [4]. Equations (12)-(13) ave the isplacements and stresses jumps at the interface in the one-order . symptotic theory. They depend on the displacements and the stresses fies 's au the zero-order and on their first and second-order derivatives. 
The hard interface law in the reference configuration (Fig.2 2 ) is derived by considering asymptotic expansions $\mathrm{A} .14 \mathrm{a}$ and $\mathrm{A} .5 \mathrm{a}$ combined with Eqs. (10)- (13) [25]:

$$
\begin{aligned}
{\left[\left[\mathbf{u}^{\varepsilon}\right]\right] \approx \varepsilon } & \left(\left(\mathbf{K}^{33}\right)^{-1}\left(\left\langle\left\langle\boldsymbol{\sigma}^{\varepsilon} \mathbf{i}_{3}\right\rangle\right\rangle-\mathbf{K}^{\alpha 3}\left\langle\left\langle\mathbf{u}_{, \alpha}^{\varepsilon}\right\rangle\right\rangle\right)-\left\langle\left\langle\mathbf{u}_{, 3}^{\varepsilon}\right\rangle\right\rangle\right) \\
{\left[\left[\boldsymbol{\sigma}^{\varepsilon} \mathbf{i}_{3}\right]\right] \approx } & \varepsilon\left(\left(-\mathbf{K}^{\beta \alpha}\left\langle\left\langle\mathbf{u}_{, \beta}^{\varepsilon}\right\rangle\right\rangle-\mathbf{K}^{3 \alpha}\left(\mathbf{K}^{33}\right)^{-1}\left(\left\langle\left\langle\boldsymbol{\sigma}^{\varepsilon} \mathbf{i}_{3}\right\rangle\right\rangle-\mathbf{K}^{\beta 3 / /} \mathbf{\imath}_{, \beta /}^{\varepsilon},\right)_{, \alpha}\right.\right. \\
& \left.-\left\langle\left\langle\boldsymbol{\sigma}_{, 3}^{\varepsilon} \mathbf{i}_{3}\right\rangle\right\rangle\right)
\end{aligned}
$$

\section{Introduction of the micro-cracking damage}

In this section, it is shown how to include micro crac ing damage in the hard interface law above obtained. The closer-f $f_{\ldots}$ of the effective elastic tensors $\mathbf{K}^{j l}$ in Eqs. (14)-(15) is specialized ho usı g micromechanical homogenization in the case of two microcracks ' macerial models: KachanovSevostianov (KS) and Welemane-Goidescu ( ${ }^{+c}$ ) models. The evolution law of the generalized microcracks density is $\mathrm{d}$ ?ruved from a thermodynamic approach and then included in the bard interface model via the asymptotic expansions method.

\subsection{Micromechanical homogen ${ }_{\text {, }}+i$ on approaches}

The Kachanov-Sevostianov . - Jel [32, 37] is a stress-based approach based on the non-interacting mi "ocr ıcks approximation [35, 36]. The WelemaneGoidescu model [40, 40, 50] is is strain-based approach, based on the dilute limit hypothesis [39] For both models, it is assumed that the material interphase comprj es a n or thotropic matrix embedding a family of microcracks parallel to $\mathbf{i}_{1}$. For u. e sake of simplicity, the formulations are reduced to 
the two-dimensional case on the plane $\left(\mathbf{i}_{1}, \mathbf{i}_{3}\right)$ with reference to the problem geometry in Fig.2.

\subsubsection{Kachanov-Sevostianov model}

Following the theory proposed by Kachanov and coworkers 32 ba id on the Eshelby's approach [51], and the above assumptions on mic ${ }^{\circ}$ racks and matrix, the interface stiffness can be derived as follows:

$$
\begin{aligned}
& K_{11}^{11}=\frac{\left(E_{1}^{0}\right)^{2}\left(2 R B_{n n} E_{3}^{0}+1\right)}{E_{1}^{0}-E_{3}^{0}\left(\nu_{13}^{0}\right)^{2}+2 R B_{n n} E_{1}^{0} E_{3}^{0}} \\
& K_{31}^{13}=K_{13}^{31}=\frac{E_{1}^{0} E_{3}^{0} \nu_{13}^{0}}{E_{1}^{0}-E_{3}^{0}\left(\nu_{13}^{0}\right)^{2}+2 R B_{\text {เn }}\left\llcorner{ }^{\top} E_{3}^{0}\right.} \\
& K_{33}^{33}=\frac{E_{1}^{0} E_{3}^{0}}{E_{1}^{0}-E_{3}^{0}\left(\nu_{13}^{0}\right)^{2}+2 R B_{\text {ง, }} \bar{L}_{1}{ }_{1} E_{3}^{-}} \\
& K_{11}^{33}=\frac{2 G_{13}^{0}}{2+R B_{t t} G_{13}^{0}}
\end{aligned}
$$

where $E_{1}^{0}, E_{3}^{0}, G_{13}^{0}, \nu_{13}^{0}$ and $\nu_{31}^{0}$ are the in-pla. slastic orthotropic moduli of the matrix; $B_{n n}$ and $B_{t t}$ are elastic paras $\_$te s depending on the matrix and microcracks characteristics [32, 33].

Note that the engineering mod ${ }^{\prime 1:}$ caı be also easily derived. The effective Young's modulus in normal d'recur $\cdot\left(1_{3}\right)$, used in the examples below, reads as:

$$
\text { П. }=\frac{E_{3}^{0}}{1+2 R B_{n n} E_{3}^{0}}
$$

\subsubsection{Welemane-Goide o \& mou ¿l}

In [40, 49, 50, 52]. Weiemane and coworkers extended the energy-based homogenization $\mathrm{dp}$ e oacn originally proposed in [39] for isotropic materials to the case of an or u. stropic matrix. 
By following the Welemane-Goidescu model [49], the expressions of the interface stiffness read as:

$$
\begin{aligned}
& K_{11}^{11}=\frac{E_{1}^{0}}{E_{3}^{0}\left(\nu_{13}^{0} \nu_{31}^{0}-1\right)^{2}}\left(E_{3}^{0}\left(1-\nu_{13}^{0} \nu_{31}^{0}\right)-R \sqrt{E_{3}^{0}}\left(\nu_{31}^{0}\right)^{2} \pi \chi\right) \\
& \left.K_{31}^{13}=K_{13}^{31}=\frac{E_{1}^{0} \nu_{31}^{0}}{\left(\nu_{13}^{0} \nu_{31}^{0}-1\right)^{2}}\left(\left(1-\nu_{13}^{0} \nu_{31}^{0}\right)-R \sqrt{E_{3}^{0}} \pi\right)\right) \\
& K_{33}^{33}=\frac{E_{3}^{0}}{\left(\nu_{13}^{0} \nu_{31}^{0}-1\right)^{2}}\left(\left(1-\nu_{13}^{0} \nu_{31}^{0}\right)-R \sqrt{E_{3}^{0}} \pi \chi\right) \\
& K_{11}^{33}=G_{13}^{0}\left(1-R \frac{\pi}{\sqrt{E_{1}^{0}}} G_{13}^{0} \chi\right)
\end{aligned}
$$

where $\chi=\left(\frac{1}{G_{13}^{0}}-2 \frac{\nu_{13}^{0}}{E_{1}^{0}}+\frac{2}{\sqrt{E_{1}^{0} E_{3}^{0}}}\right)^{\frac{1}{2}}$, and $E_{1}^{0}, E_{3}^{0}, C_{i 13}, \quad{ }_{13}^{0}$ and $\nu_{31}^{0}$ are the in-plane elastic orthotropic moduli of the matrix.

Also in this case, the engineering moduli can ${ }^{\prime}$ derived. The effective Young's modulus in normal direction $\left(\mathbf{i}_{3}\right)$, adopte ' for next examples below, reads as:

$$
E_{3}=E_{3}^{0}\left(1-2 R_{\Lambda_{\perp . \curlywedge}} E_{3}^{0}\right)
$$

with $H_{n n}$ an elastic parameter depeading on the matrix and microcracks characteristics [49] (analogous to tho pà ameter $B_{n n}$ of the KS model).

\subsection{Damage evolution law}

The proposed hard interf: - law expressed by Eqs. 12 - -13 in the limit configuration (Fig. 22), or by Iqs. (14)-(15) in the reference configuration (Fig. 2a), depends on tr. jentı ized microcracks density $R$ via the effective stiffness tensors expre ste oy Eqs. (16) and Eqs. (18) for the KS and WG model, respectiv', 1 .

A possible evolu ${ }^{\circ}$ law of $R$ in the interphase $\mathcal{B}^{\varepsilon}$ (of thickness $\varepsilon$ ) is herein derived following a thermodynamic approach [6, 7]. A pseudo-potential of 
dissipation $\Phi$ given by the sum of a quadratic term and a positively 1homogeneous functional is considered [7]. The dissipative character of the evolution of damage is given by the rate-dependent form of the poter ial:

$$
\Phi(\dot{R})=\frac{1}{2} \eta^{\varepsilon} \dot{R}^{2}+I_{[0,+\infty[}(\dot{R}),
$$

where $\eta^{\varepsilon}$ is a positive viscosity parameter; $I_{\mathcal{A}}$ denotes the indj satc furction of the set $\mathcal{A}$, i.e. $I_{\mathcal{A}}(x)=0$ if $x \in \mathcal{A}$ and $I_{\mathcal{A}}(x)=+\infty$ otharwse; $\dot{R}$ is the increment of microcracks density compared to its initial leveı, indicated in what follows as $R_{0}$. The term $I_{[0,+\infty[}(\dot{R})$ forces $\dot{R}$ to sume non-negative values and it gives the irreversible character of the d دgra 'ation process for a non-regenerative microcracked material $\left(R \geq R_{0}\right)$

The free energy associated with the constitratu a quation of the microcracked material is chosen as follows:

$$
\left.\Psi\left(\mathbf{e}\left(\mathbf{u}^{\varepsilon}\right), R\right)=\frac{1}{2} \mathbb{B}^{\varepsilon}(R)\left(\mathbf{e}\left(\mathbf{u}^{\varepsilon}\right): \boldsymbol{r}^{\varepsilon}\right)\right)-\omega^{\varepsilon} R+I_{\left[R_{0},+\infty[\right.}(R)
$$

where $\mathbb{B}^{\varepsilon}(R)$ is the effective stiffness tənsor of the material (obtained via the KS or WG model); $\mathbf{u}$ is the displac ${ }^{-n}$ en the small perturbation hypot ${ }^{\text {hesis, }},{ }^{\varepsilon}$ is a strictly negative parameter. Note that the irreversible character ,i da rage, already imposed in Eq. (20), allows to neglect the term $\left.I_{\left[R_{0},+\infty^{\prime \prime}\right.}\right)_{1: 1}$ Eq. (21).

By deriving Eqs. (20) a. $\sqrt{21}$ with respect $\dot{R}$ and $R$ respectively, then by replacing them intc the movement equations in $\mathcal{B}^{\varepsilon}$ (for further details refer to [6, 53]), the ${ }_{1}$ "'owing damage evolution law for $\dot{R}$ in the volume $\mathcal{B}^{\varepsilon}$ is obtained:

$$
\eta^{\varepsilon} I_{\iota}=\left(\omega^{\varepsilon}-\frac{1}{2} \mathbb{B}_{, R}^{\varepsilon}(R)\left(\mathbf{e}\left(\mathbf{u}^{\varepsilon}\right): \mathbf{e}\left(\mathbf{u}^{\varepsilon}\right)\right)\right)_{+}
$$


where $(\cdot)_{+}$denotes the positive part of the function and $\mathbb{B}_{, R}^{\varepsilon}(R)$ indicates the component-wise derivative of the stiffness tensor with respect to the generalized microcracks density $R$.

\subsubsection{Asymptotic theory}

In this section, the asymptotic behavior of the volumetric damas srolution law (Eq. (22)) is studied. It is prescribed that $\eta^{\varepsilon}$ and $\omega^{\varepsilon}$, a volumetric densities and thus they are inversely proportional to the 'nn dimensional interphase thickness $\varepsilon$ : $\eta^{\varepsilon}=\eta \varepsilon^{-1}$ and $\omega^{\varepsilon}=\omega \varepsilon^{-1}$, wit $\eta>0$ and $\omega<0$. Subsequently, for the sake of simplicity, we will further ass me that $\omega$ and $\eta$ do not depend on the direction orthogonal to the inte carr, surface $x_{3}$ (respec-

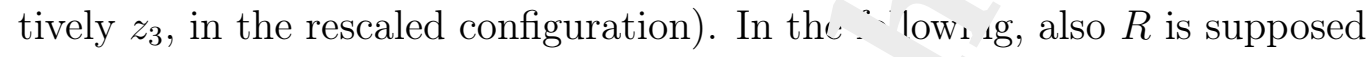
to be independent of $x_{3}$ (respectively $z_{3}$ ).

Let focus on the term: $\frac{1}{2} \mathbb{B}_{, R}^{\varepsilon}(R)\left(\mathbf{e}\left(\mathbf{u}^{\varepsilon}\right): \mathbf{e}\left(\mathbf{u}^{\prime \prime}\right)\right.$ in Eq. $(22)$. This term can be developed at 0 -order as $\frac{1}{2} \mathbb{B}_{, R}^{\varepsilon}(R)\left(\hat{\mathbf{e}}^{0} \Omega^{\urcorner}\right.$? and the constitutive equation $4 \mathrm{~b}$ leads to $\frac{1}{2} \mathbb{B}_{, R}^{\varepsilon}(R)\left[\left(\mathbb{B}^{\varepsilon}\right)^{-1}(R) \hat{\sigma}^{0}: \hat{\mathbf{e}}^{0}\right]$. Note that:

$$
\hat{\mathbf{e}}^{0}=\operatorname{Sym}\left(\hat{u}_{, 1}^{0} \otimes_{\nu_{1}}\left\llcorner^{\prime} i_{, 2}^{u} \otimes i_{2}+\hat{u}_{, 3}^{1} \otimes i_{3}\right)\right.
$$

where Sym gives the symmetrir $\mu^{+}+$of the enclosed tensor. This term is integrated along $z_{3}$ and gives $\frac{1}{2} \mathbb{B}_{\left.\left.R{ }^{\varepsilon}\right\urcorner^{\neg}\right)}^{\neg}\left[\left(\mathbb{B}^{\varepsilon}\right)^{-1}(R) \hat{\sigma}^{0}:\left\langle\hat{\mathbf{e}}^{0}\right\rangle\right]$ or $\frac{1}{2} \mathbb{B}_{, R}^{\varepsilon}(R)\left(\hat{\mathbf{e}}^{0}:\left\langle\hat{\mathbf{e}}^{0}\right\rangle\right)$. Next, by integrating agaij. $\operatorname{lor} g z_{3}$, it gives $\frac{1}{2} \mathbb{B}_{, R}^{\varepsilon}(R)\left(\left\langle\hat{\mathbf{e}}^{0}\right\rangle:\left\langle\hat{\mathbf{e}}^{0}\right\rangle\right)$, where

$$
\left\langle\hat{\mathbf{e}}^{0}\right\rangle-_{\nu^{2}} \cdot n\left(\hat{u}_{, 1}^{0} \otimes i_{1}+\hat{u}_{, 2}^{0} \otimes i_{2}+\left[\hat{u}^{1}\right] \otimes i_{3}\right)
$$

Finally, by ador 'ing the following approximation:

$$
\operatorname{Sym}\left(\hat{u}_{, 1}^{0} \otimes i_{1}+\hat{u}_{, 2}^{0} \otimes i_{2}+\left[\hat{u}^{1}\right] \otimes i_{3}\right) \approx \operatorname{Sym}\left(\hat{u}_{, 1}^{\varepsilon} \otimes i_{1}+\hat{u}_{, 2}^{\varepsilon} \otimes i_{2}+\frac{1}{\varepsilon}\left[\hat{u}^{\varepsilon}\right] \otimes i_{3}\right)
$$


the (internal) damage evolution equation reads:

$$
\eta \dot{R}=\left\{\omega-\frac{1}{2} K_{, R}^{\varepsilon}(R)\left(\begin{array}{c}
\left\langle u_{, 1}^{\varepsilon}\right\rangle \\
\left\langle\hat{u}_{, 2}^{\varepsilon}\right\rangle \\
{\left[\hat{u}^{\varepsilon}\right]}
\end{array}\right) \cdot\left(\begin{array}{c}
\left\langle\hat{u}_{, 1}^{\varepsilon}\right\rangle \\
\left\langle\hat{u}_{, 2}^{\varepsilon}\right\rangle \\
{\left[\hat{u}^{\varepsilon}\right]}
\end{array}\right)\right\}_{+}
$$

where

$$
K^{\varepsilon}=\left(\begin{array}{ccc}
\varepsilon K^{11} & \varepsilon K^{12} & K^{13} \\
\varepsilon K^{12} & \varepsilon K^{22} & K^{23} \\
K^{13} & K^{23} & \frac{1}{\varepsilon} K^{33}
\end{array}\right)
$$

By introducing the matching conditions of the hard $i^{r^{\prime}}-r$ fa e law (Eqs. (14)(15) and neglecting the second-order terms, the finc ${ }^{\prime}, r m$ of the proposed damage evolution law for a hard interface mout. nad:

$$
\eta \dot{R}=\left\{\omega-\frac{1}{2} K_{, R}(R)\left(\begin{array}{cccc}
\left\langle\left\langle u_{, 1}^{\varepsilon}\right\rangle\right\rangle & \text { I } & \left\langle\left\langle u_{, 1}^{\varepsilon}\right\rangle\right\rangle \\
\left\langle\left\langle u_{, 2}^{\varepsilon}\right\rangle\right\rangle & \mid & \text { I } & \left\langle\left\langle u_{, 2}^{\varepsilon}\right\rangle\right\rangle \\
{\left[\left[u^{\varepsilon}\right]\right]+\varepsilon\left\langle\left\langle u_{, 3}^{\varepsilon}\right\rangle\right.} & \text { I } & {\left[\left[u^{\varepsilon}\right]\right]+\varepsilon\left\langle\left\langle u_{, 3}^{\varepsilon}\right\rangle\right\rangle}
\end{array}\right)\right\}_{+}
$$

\subsection{Connection of the generalized racks density with normalized damage} parameters

In the classical continuum di nagt theory at least one normalized damage variable is adopted to describ non-localized damage [6, 53, 54]. The simplest relationship to describe mi $\mathrm{ue}_{\mathbf{2}}$ l properties degradation is $E=E^{0}(1-D)$, where $E^{0}$ is the Young'r nou '"s of the undamaged material and $D$ is the damage variable going $f_{1} \ldots$. 0 in undamaged conditions to 1 in fully damaged conditions. This a lag description is generally used in commercial software for finite element alysis (FEA). Connection relationships between $D$ and the generalized cracks density $R$ can be obtained for both KS and WG model 
by using Eq.(17) and Eq.(19), respectively, and they read as:

$$
\begin{aligned}
& D=\frac{2 R B_{n n} E^{0}}{1+2 R B_{n n} E^{0}} \quad \text { for } K S \text { model } \\
& D=2 R H_{n n} E^{0} \quad \text { for } W G \text { model }
\end{aligned}
$$

Equations (28) show that in undamaged conditions $D=R=$ ) fo. both damaged-material models. Instead, in fully damaged conditj $\Lambda_{1 .}\left(L_{=1}\right)$, $R \rightarrow+\infty$ for the KS model and it is bounded by the value $n-1 / 2 H_{n n} E^{0}$ for the WG model. Note that to have a upper bound for ' , in the WG model, is consistent with the dilute limit theory, on wh the WG model is based [40], meaning that the model is valid for sma' ${ }^{2}$ 'sity values. These connection relationships (28) have a twofold advanuoge: (i) they allow a microstructural interpretation of the damage val . ㄴe $D$, by making explicit its dependency on material and microcracks prop ' ' 1 es; (ii) they are expected to simplify the implementation of the propo $\gamma$ ir uerface model in commercial FEA-software for future validation with n . . ical simulation.

\section{Numerical examples}

Hereafter, two academic exar $\perp_{\perp}$ 'os are used to illustrate the constitutive and structural behavior of th $\mathrm{p}_{\boldsymbol{1}}$, josed hard interface model with microcracking damage. All the num ical computations have been carried out using the commercial soft. re Mathematica [55].

\subsection{0-D example: $\mathrm{Tr}$ ? s. stitutive behavior}

In this sectio.., $0-1$ example is developed to illustrate the constitutive behavior of the int. 'se model. Different points are discussed: the comparison between damaged material models KS and WG; the influence of damage 
parameters $\eta$ and $\omega$ on the interface law; and finally, the influence of the loading rate and of cyclic loads on the interface behavior.

\subsubsection{Effects of the damage evolution law}

The mechanical properties of the damaged material (Young', .. ' du' ds $\left.E_{d}\left(E_{u}, R\right)\right)$ in the case of KS (Eq. (17)) and WG (Eq. (19)) morels, nd as follows:

$$
\begin{aligned}
& E_{d}^{K S}\left(E_{u}, R\right)=\frac{E_{u}}{1+2 \pi R} \quad \text { for } K S \text { model } \\
& E_{d}^{W G}\left(E_{u}, R\right)=E_{u}(1-2 \pi R) \quad \text { for WC model }
\end{aligned}
$$

where $B_{n n}=H_{n n}=\frac{\pi}{E_{u}}$. By deriving with respect $R$ ont obtains:

$$
\begin{aligned}
& \left(E_{d}^{K S}\right)_{, R}=-\frac{2 \pi E_{u}}{(1+2 \pi R)^{2}} \quad \text { Ju. KS model } \\
& \left(E_{d}^{W G}\right)_{, R}=-2 \pi E_{u} \quad \text { for } \text { WG. odel }
\end{aligned}
$$

The damage evolution laws in the 0-D case, " both KS and WG models, are obtained substituting Eqs. (30) into 31.27 ):

$$
\eta \dot{R}= \begin{cases}\left(\omega-\frac{1}{2} \frac{\left(E_{d}^{K S}\right)}{\varepsilon} \cdot\left\ulcorner_{\imath,}\right]_{\imath}^{2}\right)_{+} & \text {for KS model } \\ \left(\omega-\frac{1}{2} \frac{\left(E_{d}^{W}{ }^{i}\right)_{,}}{\varepsilon}[u]_{n}^{2}\right)_{+} & \text {for WG model }\end{cases}
$$

Equations (31) have been nu... rrically solved with an imposed displacement jump equal to $[u]_{n}=[u]_{m c}{\overline{\digamma_{f}}}_{\text {rith }}[u]_{\max }=0.1 \mathrm{~mm}$ and $t_{f}=5 \mathrm{~s}$. Note that the time unit (s) is only $y$ alita ve and the proposed model does not depend on it because the inte fau model is developed in a quasi-static framework. Moreover, let $E_{\iota}-70<10^{3} \mathrm{MPa}$ and $\varepsilon=2 \mathrm{~mm}$. The chosen reference values for damage ${ }_{r}{ }^{r}$ imeters are $\eta=30 \mathrm{MJ} . \mathrm{s} / \mathrm{mm}^{2}$ and $\omega=-2 \mathrm{MJ} / \mathrm{mm}^{2}$. Initial damage was imposed to vanish $\left(R_{0}=0\right)$. To investigate the effects 


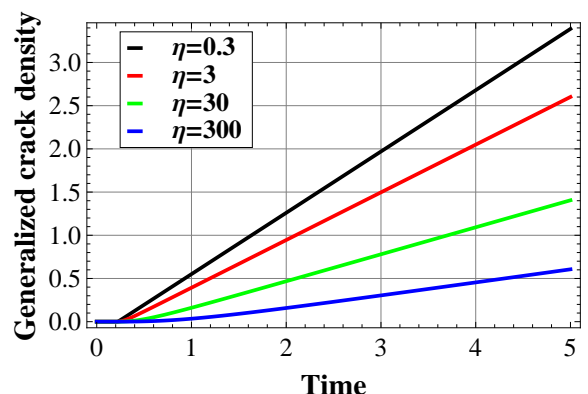

(a)

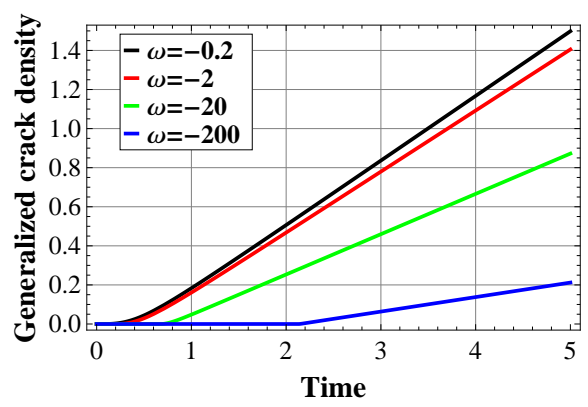

(c)

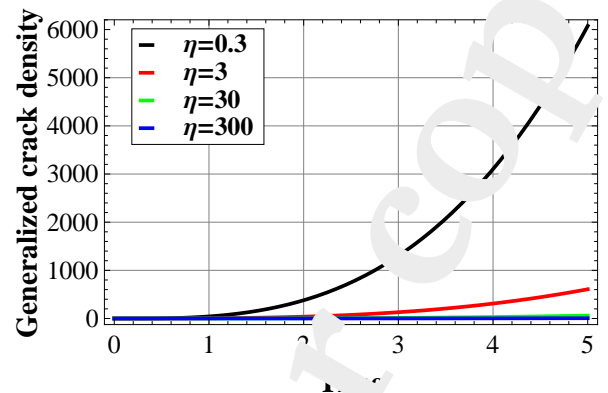

(1)

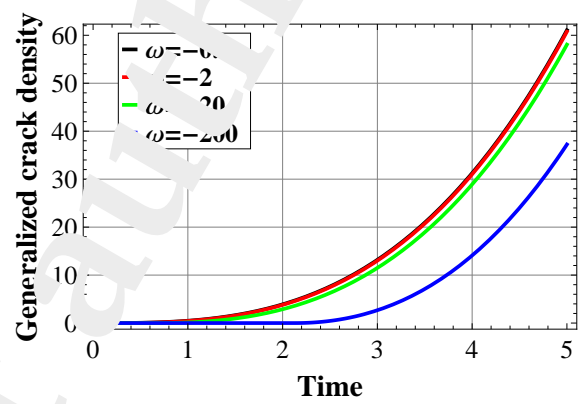

(d)

Fig. 3: Evolution of the ge 'era. 'ed microcracks density R. Fig. 3(a) effect of varying $\eta$ in the $\mathrm{KS}$.. $_{\text {. }}$ del. Fig. 3(b). effect of varying $\eta$ in the WG model. Fig. 3(c), eff, of varying $\omega$ in the KS model. Fig. 3(d); effect o r ar ing $\omega$ in the WG model.

Figures $3 \mathrm{a}-\mathrm{d}$ s. $\mathrm{w}$ t s.e evolution of the generalized microcracks density $R$ as a function $\mathrm{O}_{\perp} \mathrm{h}_{\lrcorner}$time and of damage parameters $\eta$ and $\omega$, for both KS and WG models. At the beginning, both models present an horizontal 
plateau at zero (because of the imposed initial damage $R_{0}=0$ ); then, after damage initiation, a linear increasing behavior is found for the KS model and a cubic increasing behavior for the WG model. An inverse proportir nality between $R$ and $\eta$ is found in both models (see Fig. 3(a) and Fig. 3, 3 , , 'his highlights that $\eta$ has the physical meaning of a damage viscosity $n f l u$ ncing the velocity (slope of $(R, t)$ curves) of the damage evolution. 11. res alt is also emphasized in Fig. 4, where the degradation of the $\mathrm{V}$ ang $\mathrm{s}$ modulus of both damaged materials $\mathrm{KS}$ and $\mathrm{WG}$ is shown. The slope of $\left(E_{d} / E u, t\right)$ curves, for both KS and WG models, increases as $\eta$ der aases, meaning that material get damaged "faster" for smaller values of $\%$

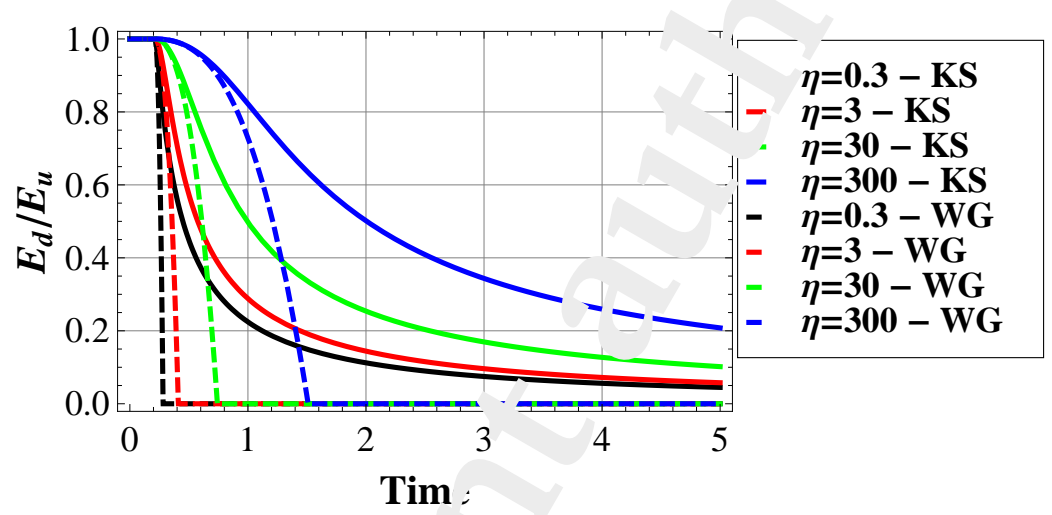

Fig. 4: Evolution in time of t' 3 1. ung's modulus of the damaged materials: parametric study on $\eta$. Kachano, jevostianov (KS, solid lines) and WelemaneGoidescu (WG, dashed line, dar saged material models are represented.

The parameter " n w the physical meaning of a threshold energy beyond which damage in. 'te. in analogy with Dupré's energy for adhesion [53]. In fact, the damage-initiation time, i.e., when $R$ begins to increase, is more 
influenced by $\omega$ than by $\eta$ for both damaged materials, as highlighted in Figs. 3(c), 3(d) and 5 .

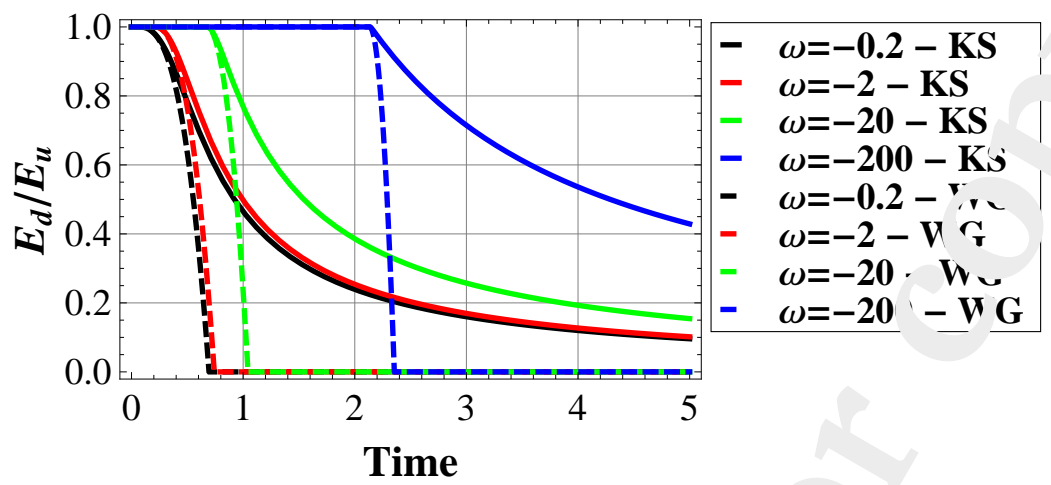

Fig. 5: Evolution in time of the Young's modulus of th - dinnaged materials: parametric study on $\omega$. Kachanov-Sevostianov model ( $\mathrm{K}_{\sim}$, ${ }^{\circ} \mathrm{l}_{1} d$ lines) and WelemaneGoidescu model (WG, dashed lines).

Moreover, Figs. 4 and 5 show that t \& crmplete damage (i.e., when $E_{d}$ tends to zero) occurs earlier for the WG model than for the KS model independently of $\omega$ and $\eta$. Note that $\mathrm{j}^{-}$the case of KS model, $E_{d}$ tends to zero asymptotically (data not shov" consistent with the two differe it hy potheses on which the models are based. Particularly, WG model is $\cdots \cdots \mathrm{d}$, n the dilute limit hypothesis, meaning that it is valid for small density Thes (less than $20 \%$ according to [39]). This is also in agreement with $r_{\text {.e }} f_{\text {ict }}$ that the generalized cracks density $R$ has an upper bound in the,- of WG model (see Section 3.3). The KS model is

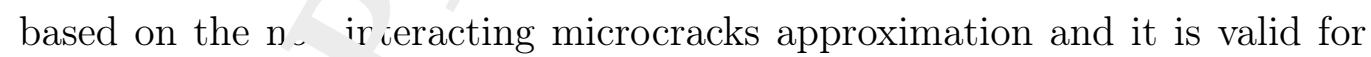
greater microcracks cuensities (until $80 \%$ according to [36, 37]). For further 

by Eqs. 31 .

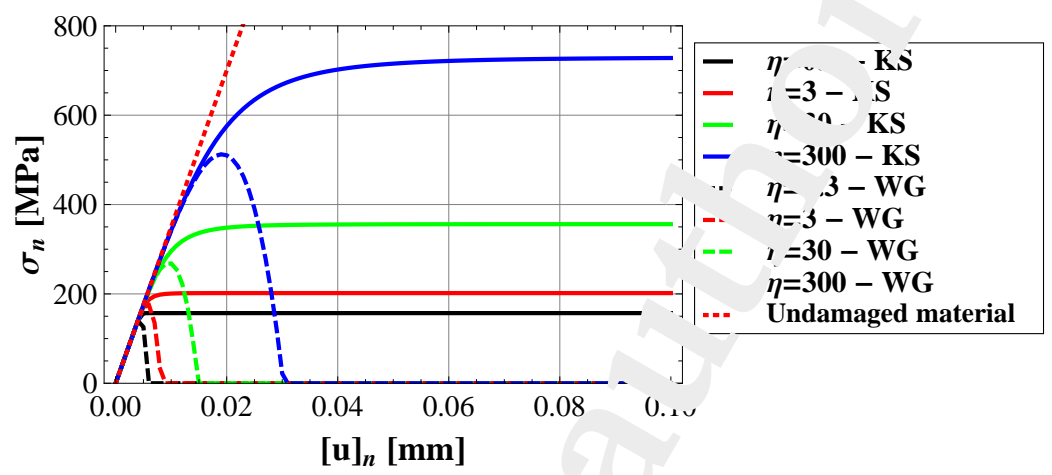
reader can refer to [37.

The interface model in $0-\mathrm{D}$ can be expressed as:

$$
\sigma_{n}=\frac{E_{d}}{\varepsilon}[u]_{n}
$$

Equation (32) has been solved for both KS and WG models, rf pla $\operatorname{ng} \pm_{d}$ by $E_{d}^{K S}$ and $E_{d}^{W G}$, respectively (see Eqs. (29)), in which $R$ hrs been obtained

Fig. 6: Interface law: parametric stui on $\eta$. Kachanov-Sevostianov (KS, solid lines) and Welemane-Goidescu (WG « rhed lines) damaged material models are represented. The linear-elastic ahavic of the undamaged material is represented with a red dotted line.

321

322

details regarding the difference between these microstructural hypotheses the 


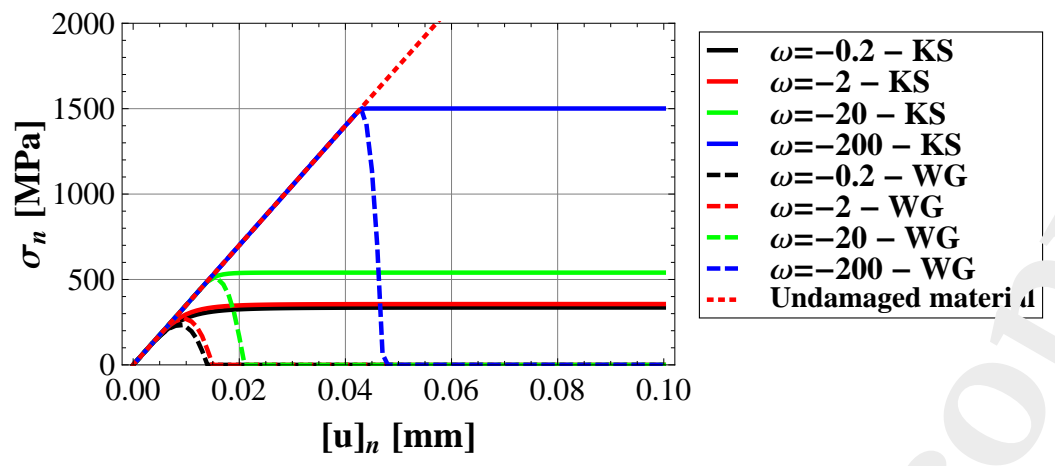

Fig. 7: Interface law: parametric study on $\omega$. Kachanov-Sevostranov (KS, solid lines) and Welemane-Goidescu (WG, dashed lines) damag material models are represented. The linear-elastic behavior of the undamag $: \mathrm{d}_{\perp}$ iterial is represented with a red dotted line.

elastic limit increases with $\eta$ and this result _ nfirms the role of the damage viscosity $\eta$ as the velocity of the damage evolun $\mathrm{n}$. Figure 6 shows also that $\eta$ influences the nonlinear transition betwe or the linear elastic domain and the damaged domain (this is more evident in KS model than in WG model); thus for a small damage viscosity $\eta$ this ' 'nsıion tends to vanish (i.e., suggesting that the material gets damą $d$ in. "diately after the initiation). Figure 7 emphasizes the role of paramı wer $w$ as a damage initiation threshold: thus the higher is $\omega$, the later $r^{\prime}$ vage initiates (see Fig. 5) and the higher the elastic limit.

\subsubsection{Effects of the 1, ading rate}

The influencr of he luading rate and of the loading shape on the interface model has been inve igated. In particular, two displacement jumps have 

$70 \times 10^{3} \mathrm{MPa}, \varepsilon=2 \mathrm{~mm}$ and $R_{0}=0$, are taken as in the $\mathrm{p}$. vious study. $[u]_{n}=v t$ and a quadratic function $[u]_{n}=1 / 2 v^{2} t^{2}+1 / 2 v t$. Four values of the loading rate $v=[u]_{\max } / t_{f}$ have been simulated $(0.1,0.2,2,20) \mathrm{mm} / \mathrm{s}$ vith a fixed $[u]_{\max }=1 \mathrm{~mm}$ and by varying the duration $t_{f}$ between $\left(0.05,6 . v, v,{ }^{1} 0\right)$ s. The damage parameters have been taken equal to their refer nce values $\eta=30 \mathrm{MJ} . \mathrm{s} / \mathrm{mm}^{2}$ and $\omega=-2 \mathrm{MJ} / \mathrm{mm}^{2}$. The other pars me rs $\unlhd_{u}=$
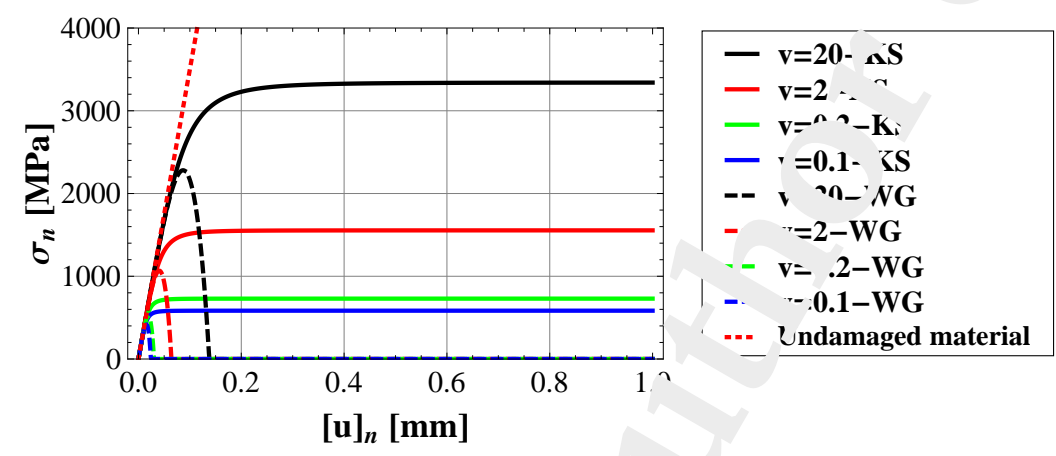

Fig. 8: Interface law for a ramp displace. if nt jump: parametric study on the loading rate $v$. Kachanov-Sevostianov (KS, solid lines) and Welemane-Goidescu (WG, dashed lines) damaged material mu sls are represented. The linear-elastic behavior of the undamaged materic . $*$ represented with a red dotted line.

been separately imposed to solve Eqs. (32), 29), and (31): a ramp function

Figure 8 shows the int .. "e law in the case of the ramp displacement jump. In analogy with tho pı - ious section, a ductile damage behavior of the

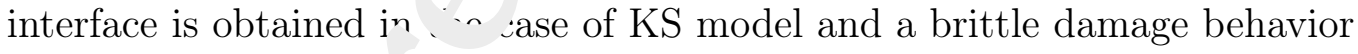
for WG model.

Figure 9 show "ie interface law in the case of the quadratic displacement jump. The imposed quadratic displacement jump produces an hardening-like 


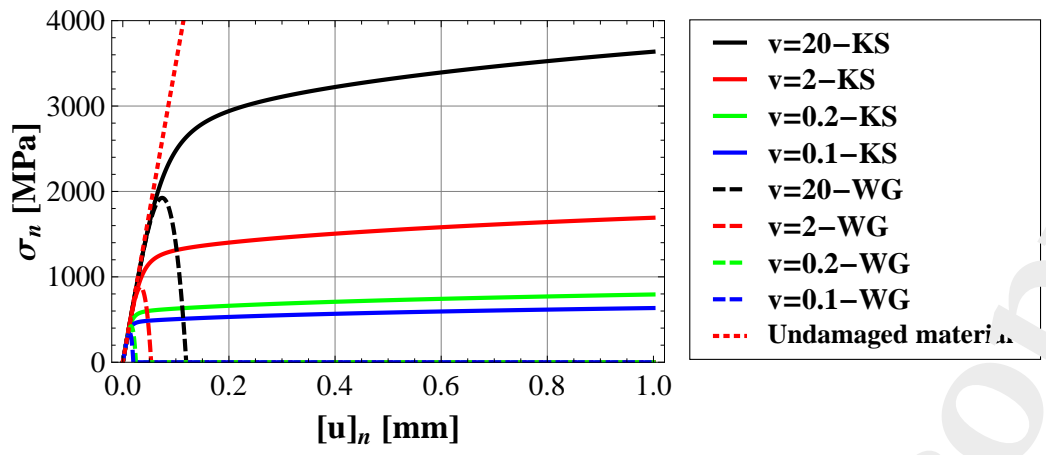

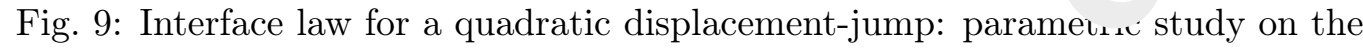
loading rate $v$. Kachanov-Sevostianov (KS, solid lines) ar-Welemane-Goidescu (WG, dashed lines) damaged material models are reprer, «. $\mathrm{d}$. The linear-elastic behavior of the undamaged material is represented with a $\mathrm{d}$ dotted line.

effect in the damaged part of the interface cor tituuve behavior (i.e., beyond the elastic limit) and the slope increases $\mathrm{w}_{\iota}{ }^{+1}$ e loading rate $v$.

Both Figs. 849 highlight that for hig] rau $(v=2,20 \mathrm{~mm} / \mathrm{s})$ the elastic limit (tensile) is higher than in the arasi-static configurations $(v=0.1,0.2$ $\mathrm{mm} / \mathrm{s}$ ) for both KS and WG modela. hueently, authors provide a validation of the proposed hard interface mo ${ }^{\prime}$ in [31], by comparing simulated response curves with data from tensile $x$ x mental tests available in the literature [56] in both quasi-static and hly. -rate loading conditions. They found that the loading-rate depender.. of the hard interface model makes it suitable to describe the experimen $7^{\prime}$ ' b' havior observed in [56].

\subsubsection{Effects of $-{ }^{7} i c$ « ading}

The influence $u_{\perp}$ velic loading on the hard interface model has also been investigated. A strictly positive sinusoidal displacement jump has been im- 

$\mathrm{MPa}, \varepsilon=2 \mathrm{~mm}$ and $R_{0}=0$, as in the previous study.

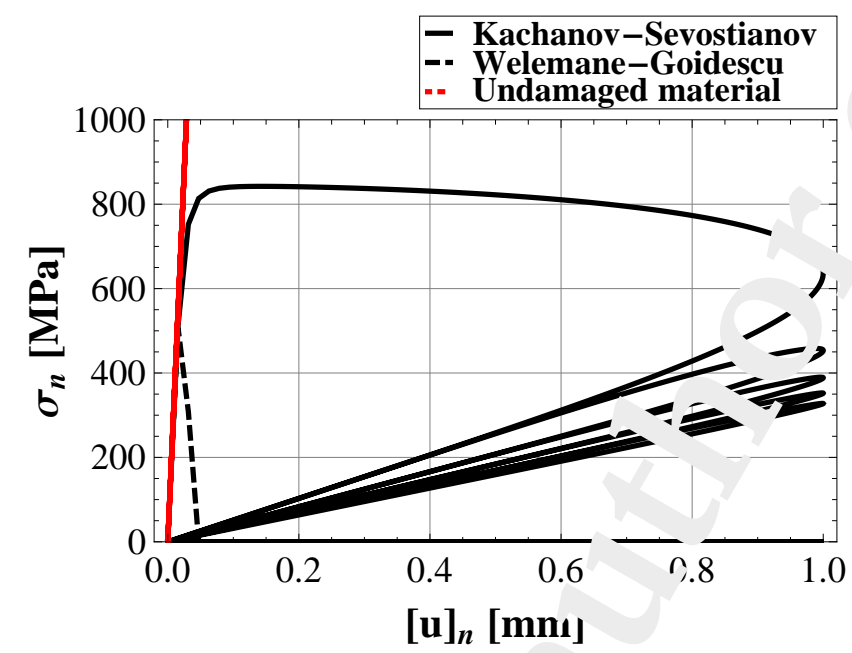

Fig. 10: Interface law for a cyclic load for KS und WG model. The linear-elastic

behavior of the undamaged material is . nre sented with a red dotted line.

posed: $[u]_{n}=[u]_{\max }\left|\sin \left(f t / t_{f}\right)\right|$, with $[u]_{\max }=1 \mathrm{~mm}, f=\pi / 2, t_{f}=5 \mathrm{~s}$ and 5 cycles have been considered. Both KS and WG damage models have been considered and the damage parameters have been taken equal t $\mathrm{t}$ their reference values $\eta=30 \mathrm{MJ} . \mathrm{s} / \mathrm{mm}^{2}$ and $\omega=-2 \mathrm{MJ} / \mathrm{mm}^{2} . E_{u}=1 \mathrm{u} \wedge .10^{3}$

As shown in Fig. 10, the $t^{\circ} \mathrm{O}$. . mage models give very different results under the same loading and nar neter conditions. KS model, together with the proposed damage evo' '

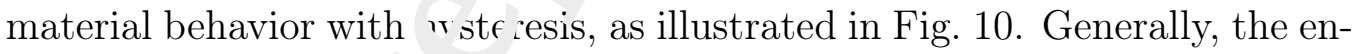
ergy dissipated via $\mathrm{m}{ }^{*}{ }^{\mathrm{r}} \mathrm{O}$-cracking damage is higher at the initiation and first accumulation of nic ocracks. This is consistent with the resulting hysteresis loop of the first cycle that is larger than the others; after the first cycle, the 
hysteresis decreases with the number of cycles until the damage evolution is completed. Moreover, the damage evolution produces a decreasing of the interface stiffness (see Fig. 10). The stiffness of the undamaged mat rial is equal to $35000 \mathrm{~N} / \mathrm{mm}^{3}$ and after the first cycle it reduces to $515 \mathrm{~N} / \mathrm{mm}_{\mathrm{n}} \mathrm{z}^{3}$. After the first reloading (2nd cycle), the stiffness slightly decreas * un il the damage evolution is completed, and at the end of the fifth cyc'e $\iota_{\nu_{2}}$ ? stı.fness is equal to $318 \mathrm{~N} / \mathrm{mm}^{3}$. This result is physically plausible.

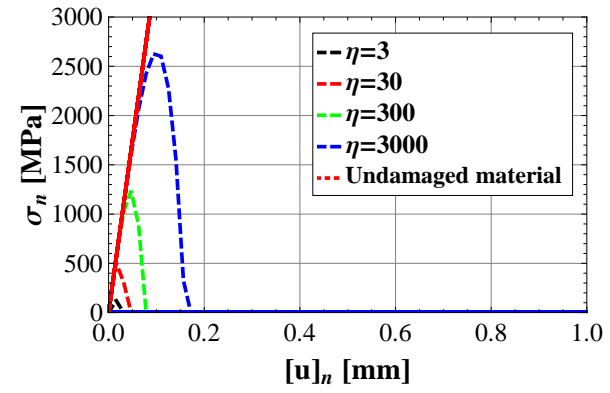

(a)

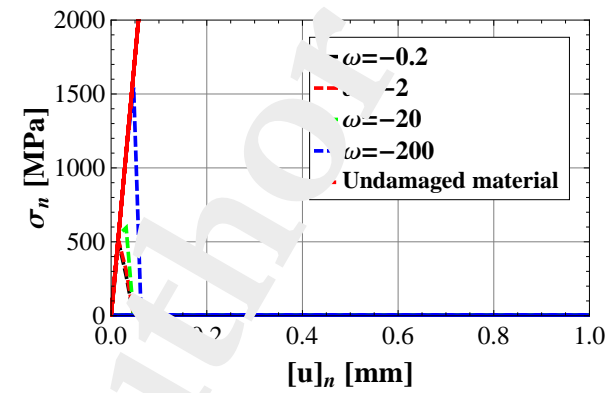

(b)

Fig. 11: Interface law for a cyclic load for WG model. Fig. 11(a): study on $\eta$. Fig. 11(b) study on $\omega$.

On the contrary, WG dama ${ }_{c}$ ' mudel is not able to reproduce a damage behavior under cyclic loads. '? rure 10 shows an abrupt reduction in stiffness to zero already during the th ; loading curve, meaning that the damaged material behavior is br: ${ }^{\prime}$ e, $\iota_{\llcorner}$tgreement with the previous results. Note that this behavior de $\mathrm{s}$..ut depend on the chosen values of the damaged parameters $\eta$ an $i_{\text {in }}$ as slustrated in Fig. 11 .

Finally, Fig. 1\%. ' ws the evolution in time of the normal stress $\sigma_{n}$ in the case of KS model, highlighting the decrease of the maximum normal stress 
with the number of cycles (note a decrease of the $60 \%$ at the last cycle).

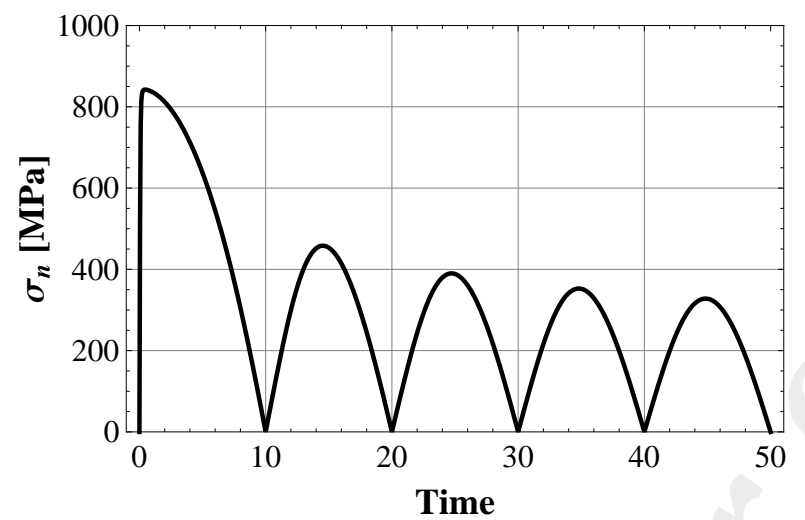

Fig. 12: Interface law for a cyclic load: normal stress as a in ction of the time for the Kachanov-Sevostianov damage model.

\subsection{1-D example: The structural behavior}

In this section, a simple 1-D example is u veloped to illustrate the structural behavior of the proposed hard interface model. A composite bar under traction was considered. The bar, of see ' on $A$, comprised two parts of length $\ell$, made of an undamaged materic 'vith. Young's modulus $E_{u}$, and an embedded part of length $\varepsilon$, made of $z \iota^{\prime}$ 'nageable material (glue-like interphase) with Young's modulus $E_{d}\left(E_{\imath},{ }^{\prime}\right.$ '. The damageable material in the interphase is supposed to have + th $\lrcorner$ beginning the same Young's modulus of the adherents, then it degr ades as whe microcracks density $R$ evolves. The bar was fixed at one enc and a quasi-static traction force was $F(t)$ applied on the other end, a : illi strated in Fig. 13 . 

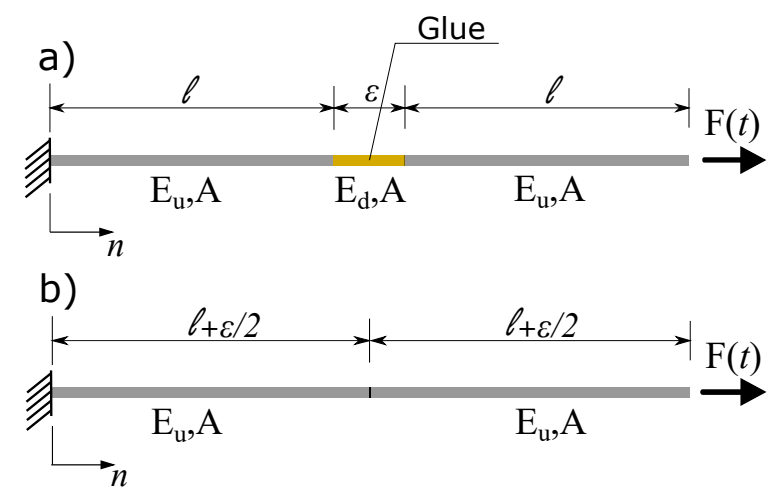

Fig. 13: 1-D example: bar under traction, a) glue interphase, b, · erface model

The displacement field can be easily derived ana'ytı liy as:

$$
u(n)= \begin{cases}\frac{F}{E_{u} A} n \quad 0 \leq n \leq \ell & \\ \frac{F}{E_{d} A} n+\frac{F \ell}{A}\left(\frac{1}{E_{u}}-\frac{1}{E_{d}}\right) & n \leq r_{\iota} \leq \ell+\varepsilon \\ \frac{F}{E_{u} A} n-\frac{F \varepsilon}{A}\left(\frac{1}{E_{u}}-\frac{1}{E_{d}}\right) & \iota+\varepsilon \leq n \leq 2 \ell+\varepsilon\end{cases}
$$

Thus, the displacement jump along $n$ is $\jmath$ ta.ned as $[u]_{n}=u(\ell+\varepsilon)-u(\ell)$ :

$$
[u]_{n}^{-}=\frac{F \varepsilon}{F_{1} A}
$$

Note that, being $\frac{F}{A}=\sigma_{n}$, the sta 1da. ? spring-like interface law in 1-D approximation can be derived (in nalcgy with Eq. (32)).

The Young's modulus of $\mathrm{t}_{\llcorner}$- damaged material $E_{d}\left(E_{u}, R\right)$ was specialized to the case of KS and $W_{\mathcal{G}}$ L odel following Eqs. (29) as in the previous example. The expressic ... of $\iota_{\ldots}$, evolution of damage Eqs. (31) taking into account the displacen $n_{\iota}$, ump Eq. (34) is derived in this 1-D case as:

$$
\dot{R}=\left\{\begin{array}{l}
\frac{-}{n}\left(\omega+\pi \frac{\sigma_{n}^{2}}{E_{u}} \varepsilon\right)_{+} \quad \text { for } K S \text { model } \\
\frac{1}{\eta}\left(\omega+\pi \frac{\sigma_{n}^{2}}{E_{u}} \varepsilon \frac{1}{(1-2 \pi R)^{2}}\right)_{+} \text {for WG model }
\end{array}\right.
$$



and a ductile behavior for KS material.

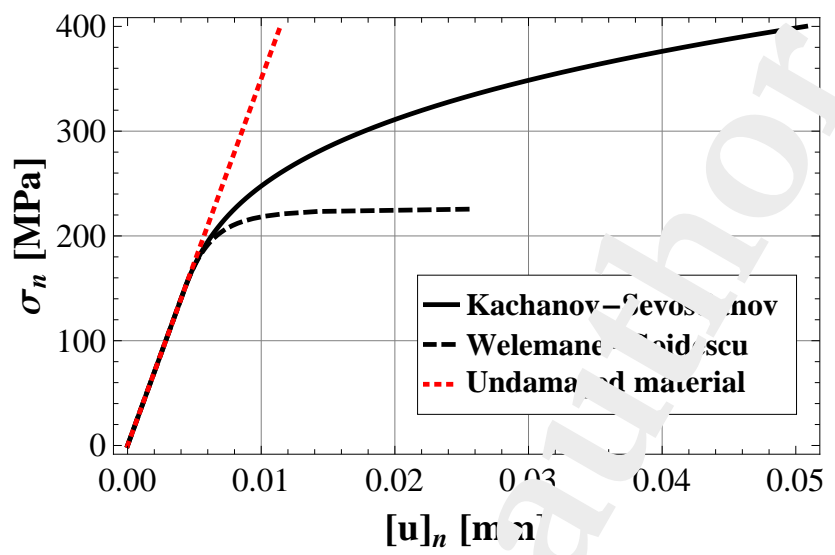

Fig. 14: Interface law in the 1-D cast Karhanov-Sevostianov model (KS, solid line), Welemane-Goidescu model ( $\mathrm{V}, \mathrm{u}$, Jashed line), undamaged material (red dotted line).

\section{Conclusions}

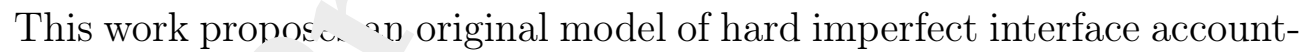
ing for micro-cre ${ }^{1}{ }^{i}{ }_{1} g$ and damage evolution. Preliminary numerical results based on simple academic examples, in terms of both constitutive and struc- 
tural behavior, are promising. They suggest that the model could represent a suitable strategy for a macroscopic description of hard adhesives with micro-cracking damage, regardless of whether they have a ductile or orittle behavior. In fact, the analytical interface model could be included in a wis ite element context via user-defined interface finite elements. Moreo ${ }^{\wedge} r$, $c$, nnection relationships between the generalized cracks density and tı sta ıdard normalized damage variable, derived at Section 3.3, are exp _teu to simplify the implementation in commercial FEA-software for future vuldation with numerical simulation.

The main perspective to enhance the proposed mu $\mathrm{el}$ is to establish a combined experimental/modelling identification $\mathrm{p}^{-\mathrm{t}}$ ocol for the damage parameters of the evolution law, the damage viscosity - and the damage threshold $\omega$. A design of experience will be set up ir or . $_{\text {. to }}$ catch the interactions between damage parameters $\eta$ and $\omega$ that $v$. $\cap$. Id only glimpse through the OFAT approach. To this aim, authors rave specialized the proposed hard interface model to the case of tubular-butt joints under combined tensiletorsion loads [31]. This is a standara perimental design used to characterize structural adhesives and $\mathrm{i}$ llows future validations of the proposed interface model with experimenta. 'ssts.

\section{A. Matched asymptot $\cdot$ ex jansions method}

\section{A.1. Rescaling phase}

The rescaling $\boldsymbol{r h}_{\mathrm{dsc}}$ if the asymptotic process represents a mathematical construct [46], il - physically-based configuration, and it is used in order to eliminate the dependency of the integration domains on the small parameter 
447

resulting

$$
\frac{\partial}{\partial z_{1}}=\frac{\partial}{\partial x_{1}}, \quad \frac{\partial}{\partial z_{2}}=\frac{\partial}{\partial x_{2}}, \quad \frac{\partial}{\partial z_{3}}=\varepsilon \frac{\partial}{\partial x_{3}}
$$

450

as well as in the adherents $\overline{\mathbf{p}}:=\left(x_{1}, x_{2}, x_{3}\right) \rightarrow\left(z_{1}, z_{2}, z_{3}\right)$ :

$$
z_{1}=x_{1}, \quad z_{2}=x_{2}, \quad z_{3}=x_{3} \pm \frac{1}{2}\left(\begin{array}{ll}
1 & -
\end{array}\right)
$$

where the plus (minus) sign applies whenever $x \in \Omega_{{ }_{1}} \prime_{i} \in \Omega^{\varepsilon}{ }_{-}$), with

$$
\frac{\partial}{\partial z_{1}}=\frac{\partial}{\partial x_{1}}, \quad \frac{\partial}{\partial z_{2}}=\frac{\partial}{\partial x_{2}}, \quad \frac{i}{\partial z_{\rho}}=\frac{\partial}{\partial x_{3}}
$$

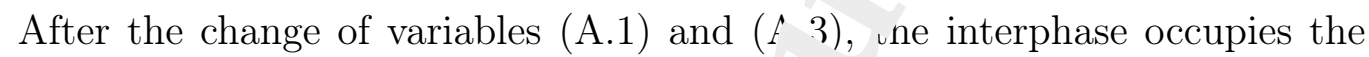
domain $\mathcal{B}=\left\{\left(z_{1}, z_{2}, z_{3}\right) \in \mathbb{R}^{3}:\left(z_{1}, z_{2}\right) \in<\left|z_{3}\right|<\frac{1}{2}\right\}$ and the adherents occupy the domains $\Omega_{ \pm}=\Omega^{\varepsilon} \pm \frac{1}{2}(1-\varepsilon)_{\mathbf{1}_{3}}$, as shown in Fig. 2 b. The sets $\mathcal{S}_{ \pm}=\left\{\left(z_{1}, z_{2}, z_{3}\right) \in \mathbb{R}^{3}:\left(z_{1}, z_{2}\right) \in \mathcal{S}_{3}= \pm \frac{1}{2}\right\}$ are taken to denote the interfaces between $\mathcal{B}$ and $\Omega_{ \pm}$and $\ell=\iota_{+} \cup \Omega_{-} \cup \mathcal{B} \cup \mathcal{S}_{+} \cup \mathcal{S}_{-}$is the rescaled configuration of the composite ${ }_{n} \gamma_{v} \cdot \Gamma_{u}$ and $\Gamma_{g}$ indicate the images of $\Gamma_{u}^{\varepsilon}$ and $\Gamma_{g}^{\varepsilon}$ after the change of vas hlos, and $\overline{\mathbf{f}}^{ \pm}:=\mathbf{f}^{ \pm} \circ \overline{\mathbf{p}}^{-1}$ and $\overline{\mathbf{g}}^{ \pm}:=\mathrm{g}^{ \pm} \circ \overline{\mathbf{p}}^{-1}$ the rescaled external force.

\section{A.2. Kinematic equatic c}

Following the . oru $\mathrm{n}$ proposed in [23, 25], let us focus on the kinematics of the elastic,$\quad$ b]em. After taking $\hat{\mathbf{u}}^{\varepsilon}=\mathbf{u}^{\varepsilon} \circ \hat{\mathbf{p}}^{-1}$ and $\overline{\mathbf{u}}^{\varepsilon}=\mathbf{u}^{\varepsilon} \circ \overline{\mathbf{p}}^{-1}$ to 
denote the displacement fields from the rescaled adhesive and adherents, respectively, the asymptotic expansions of the displacement fields with respect to $\varepsilon$ are:

$$
\begin{aligned}
\mathbf{u}^{\varepsilon}\left(x_{1}, x_{2}, x_{3}\right) & =\mathbf{u}^{0}+\varepsilon \mathbf{u}^{1}+\varepsilon^{2} \mathbf{u}^{2}+o\left(\varepsilon^{2}\right) \\
\hat{\mathbf{u}}^{\varepsilon}\left(z_{1}, z_{2}, z_{3}\right) & =\hat{\mathbf{u}}^{0}+\varepsilon \hat{\mathbf{u}}^{1}+\varepsilon^{2} \hat{\mathbf{u}}^{2}+o\left(\varepsilon^{2}\right) \\
\overline{\mathbf{u}}^{\varepsilon}\left(z_{1}, z_{2}, z_{3}\right) & =\overline{\mathbf{u}}^{0}+\varepsilon \overline{\mathbf{u}}^{1}+\varepsilon^{2} \overline{\mathbf{u}}^{2}+o\left(\varepsilon^{2}\right)
\end{aligned}
$$

with:

$$
\begin{aligned}
& \hat{\mathbf{e}}^{-1}=\left[\begin{array}{cc}
0 & \frac{1}{2} \hat{u}_{c, 3}^{0} \\
\frac{1}{2} \hat{u}_{\alpha, 3}^{0} & \hat{\iota}_{{ }_{3}}^{0}
\end{array}\right]=\operatorname{Sym}\left(\hat{\mathbf{u}}_{, 3}^{0} \otimes \mathbf{i}_{3}\right) \\
& \hat{\mathbf{e}}^{k}=\left[\begin{array}{cc}
\operatorname{Sym}\left(\hat{u}_{\alpha, \beta}^{k}\right) & \frac{1}{2}\left(\hat{u}_{3 \curlywedge}^{k}+u_{\kappa, 3}^{-1}\right) \\
\frac{1}{2}\left(\hat{u}_{3, \alpha}^{k}+\hat{u}_{\alpha, 3}^{k+1}\right) & \wedge_{3}^{k+}
\end{array}\right]=\operatorname{Sym}\left(\hat{\mathbf{u}}_{, 1}^{k} \otimes \mathbf{i}_{1}+\hat{\mathbf{u}}_{, 2}^{k} \otimes \mathbf{i}_{2}+\hat{\mathbf{u}}_{, 3}^{k+1} \otimes \mathbf{i}_{3}\right)
\end{aligned}
$$

Interphase. The gradient of the displacement field $\hat{\mathbf{u}}^{\varepsilon}$ rea,ds:

$$
\mathbf{e}\left(\hat{\mathbf{u}}^{\varepsilon}\right)=\frac{1}{2}\left[\nabla\left(\hat{\mathbf{u}}^{\varepsilon}\right)+\nabla\left(\hat{\mathbf{u}}^{\varepsilon}\right)^{T}\right]=\varepsilon^{-1} \hat{\mathbf{e}}^{-1} \uparrow \hat{\vartheta}^{\eta}+\varepsilon \hat{\mathbf{e}}^{1}+O\left(\varepsilon^{2}\right)
$$
where $\operatorname{Sym}(\cdot)$ gives the vr metric part of the enclosed tensor and $k=0,1$, and $\otimes$ is the dyaric , "ofuct between vectors such as: $(\mathbf{a} \otimes \mathbf{b})_{i j}=a_{i} b_{j}$.

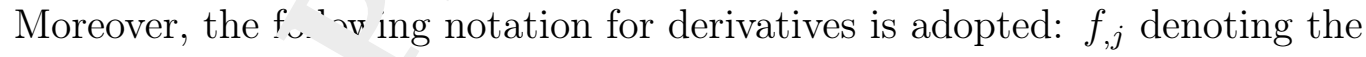
partial derivative of $j$ with respect to $z_{j}$. 
468

Adherents. The gradient of the displacement field $\overline{\mathbf{u}}^{\varepsilon}$ reads:

$$
\nabla\left(\overline{\mathbf{u}}^{\varepsilon}\right)=\left[\begin{array}{ll}
\bar{u}_{\alpha, \beta}^{0} & \bar{u}_{\alpha, 3}^{0} \\
\bar{u}_{3, \beta}^{0} & \bar{u}_{3,3}^{0}
\end{array}\right]+\varepsilon\left[\begin{array}{cc}
\bar{u}_{\alpha, \beta}^{1} & \bar{u}_{\alpha, 3}^{1} \\
\bar{u}_{3, \beta}^{1} & \bar{u}_{3,3}^{1}
\end{array}\right]+O\left(\varepsilon^{2}\right)
$$

469 So that the strain tensor is:

$$
\mathbf{e}\left(\overline{\mathbf{u}}^{\varepsilon}\right)=\frac{1}{2}\left[\nabla\left(\overline{\mathbf{u}}^{\varepsilon}\right)+\nabla\left(\overline{\mathbf{u}}^{\varepsilon}\right)^{T}\right]=\varepsilon^{-1} \overline{\mathbf{e}}^{-1}+\overline{\mathbf{e}}^{0}+\varepsilon \overline{\mathbf{e}}^{1}+O\left(\varepsilon^{-}\right)
$$

470 with:

$$
\overline{\mathbf{e}}^{-1}=\mathbf{0}
$$

471

$$
\left.\overline{\mathbf{e}}^{k}=\left[\begin{array}{cc}
\operatorname{Sym}\left(\bar{u}_{\alpha, \beta}^{k}\right) & \frac{1}{2}\left(\bar{u}_{3, \alpha}^{k}+\bar{u}_{\alpha, 3}^{k}\right) \\
\frac{1}{2}\left(\bar{u}_{3, \alpha}^{k}+\bar{u}_{\alpha, 3}^{k}\right) & \bar{u}_{3,3}^{k}
\end{array}\right]=\operatorname{Sym}_{\mathbf{1}}{ }^{k} \otimes \mathbf{1}_{1}+\overline{\mathbf{u}}_{, 2}^{k} \otimes \mathbf{i}_{2}+\overline{\mathbf{u}}_{, 3}^{k} \otimes \mathbf{i}_{3}\right)
$$

472 and $k=0,1$.

473 A.3. Equilibrium equations

The stress fields in the rescaled a " $"$ sive and adherents, $\hat{\boldsymbol{\sigma}}^{\varepsilon}=\boldsymbol{\sigma} \circ \hat{\mathbf{p}}^{-1}$ and $\overline{\boldsymbol{\sigma}}^{\varepsilon}=\boldsymbol{\sigma} \circ \overline{\mathbf{p}}^{-1}$ respectively, ca be epresented as asymptotic expansions [23, 25]:

$$
\begin{array}{rr} 
& =\boldsymbol{\sigma}^{0}+\varepsilon \boldsymbol{\sigma}^{1}+O\left(\varepsilon^{2}\right) \\
\boldsymbol{\sigma} & \hat{\boldsymbol{\sigma}}^{0}+\varepsilon \hat{\boldsymbol{\sigma}}^{1}+O\left(\varepsilon^{2}\right) \\
\boldsymbol{\sigma}^{\varepsilon}= & \overline{\boldsymbol{\sigma}}^{0}+\varepsilon \overline{\boldsymbol{\sigma}}^{1}+O\left(\varepsilon^{2}\right)
\end{array}
$$

474 Interphase. As $ר$ forces are neglected, the equilibrium equation is:

$$
\operatorname{div} \hat{\boldsymbol{\sigma}}^{\varepsilon}=\mathbf{0}
$$


475

Substituting Eq. A.14b in Eq. A.15 and using Eq. A.2, it becomes:

$$
\begin{aligned}
0 & =\hat{\sigma}_{i \alpha, \alpha}^{\varepsilon}+\varepsilon^{-1} \hat{\sigma}_{i 3,3}^{\varepsilon} \\
& =\varepsilon^{-1} \hat{\sigma}_{i 3,3}^{0}+\hat{\sigma}_{i \alpha, \alpha}^{0}+\hat{\sigma}_{i 3,3}^{1}+\varepsilon \hat{\sigma}_{i \alpha, \alpha}^{1}+O(\varepsilon)
\end{aligned}
$$

${ }_{476}$ where $\alpha=1,2$. Eq. A.16 has to be satisfied for any value of $\varepsilon$, « ti...g to:

$$
\begin{aligned}
\hat{\sigma}_{i 3,3}^{0} & =0 \\
\hat{\sigma}_{i 1,1}^{0}+\hat{\sigma}_{i 2,2}^{0}+\hat{\sigma}_{i 3,3}^{1} & =0
\end{aligned}
$$

477 where $i=1,2,3$.

478 Eq. A.17 shows that $\hat{\sigma}_{i 3}^{0}$ is not dependent on ir the adhesive, and 479 thus it can be written:

$$
\left[\hat{\sigma}_{i 3}^{0}\right]=0
$$

${ }_{480}$ where $[\cdot]$ denotes the jump between $z_{3}=\frac{1}{2}$. nnd $z_{3}=-\frac{1}{2}$. In view of 481 Eq. A.19, Eq. A.18), for $i=3$, can be 1 ritten in the integrated form

$$
\left[\hat{\sigma}_{33}^{1}\right]=\cdots j_{13,1}^{0}-\hat{\sigma}_{23,2}^{0}
$$

482 Adherents. The equilibrium equa' 'on 1.1 the adherents is:

$$
\operatorname{div} \overline{\boldsymbol{c}}^{\varepsilon}+\overline{\mathbf{f}}=\mathbf{0}
$$

483 Substituting Eq. A.14c) : Fa (A.21) that has to be satisfied for any value ${ }_{484}$ of $\varepsilon$, leads to:

$$
\begin{aligned}
& \operatorname{div} \overline{\boldsymbol{\sigma}}^{0}+\overline{\mathbf{f}}=\mathbf{0} \\
& \operatorname{div} \overline{\boldsymbol{\sigma}}^{1}=\mathbf{0}
\end{aligned}
$$




\section{A.4. Matching phase}

The imposed continuity conditions at $\mathcal{S}_{ \pm}^{\varepsilon}$ for the fields $\mathbf{u}^{\varepsilon}$ and $\boldsymbol{\sigma}^{\varepsilon}$ lead to matching relationships between external and internal expansions [23, $\div 5]$. In terms of displacements the following relationship have to be satisfiea:

$$
\mathbf{u}^{\varepsilon}\left(\mathbf{x}_{\alpha}, \pm \frac{\varepsilon}{2}\right)=\hat{\mathbf{u}}^{\varepsilon}\left(\mathbf{z}_{\alpha}, \pm \frac{1}{2}\right)=\overline{\mathbf{u}}^{\varepsilon}\left(\mathbf{z}_{\alpha}, \pm \frac{1}{2}\right)
$$

where $\mathbf{x}_{\alpha}:=\left(x_{1}, x_{2}\right), \mathbf{z}_{\alpha}:=\left(z_{1}, z_{2}\right) \in \mathcal{S}$. Expanding the displa $m$ snt in the adherents $\mathbf{u}^{\varepsilon}$, in Taylor series along the $x_{3}$-direction and tai ${ }^{\prime} \mathrm{n}$. nto account Eq. A.5a), it results:

$$
\begin{aligned}
& \mathbf{u}^{\varepsilon}\left(\mathbf{x}_{\alpha}, \pm \frac{\varepsilon}{2}\right)=\mathbf{u}^{\varepsilon}\left(\mathbf{x}_{\alpha}, 0^{ \pm}\right) \pm \frac{\varepsilon}{2} \mathbf{u}_{, 3}^{\varepsilon}\left(\mathbf{x}_{\alpha}, 0^{ \pm}\right)+\cdots \\
& =\mathbf{u}^{0}\left(\mathbf{x}_{\alpha}, 0^{ \pm}\right)+\varepsilon \mathbf{u}^{1}\left(\mathbf{x}_{\alpha}, 0^{ \pm}\right) \underset{z^{\prime}}{\varepsilon} \mathbf{l}_{3}\left(\mathbf{x}_{\alpha}, 0^{ \pm}\right)+\cdots
\end{aligned}
$$

Substituting Eqs. A.5b and A.5c together ith $\mathrm{Aq}$ (A.25) in Eq. A.24, it holds true:

$$
\begin{aligned}
\mathbf{u}^{0}\left(\mathbf{x}_{\alpha}, 0^{ \pm}\right)+ & \\
+\varepsilon \mathbf{u}^{1}\left(\mathbf{x}_{\alpha}, 0^{ \pm}\right) \pm \frac{\varepsilon}{2} \mathbf{u}_{3}^{0}\left(\mathbf{x}_{\alpha}, 0^{ \pm}\right)+\cdots & =\mathbf{u}\left(\mathbf{z}_{\alpha}, \pm \frac{1}{2}\right)+\varepsilon \hat{\mathbf{u}}^{1}\left(\mathbf{z}_{\alpha}, \pm \frac{1}{2}\right)+\cdots \\
& =\overline{\mathbf{u}}^{0}\left(\mathbf{z}_{\alpha}, \pm \frac{1}{2}\right)+\varepsilon \overline{\mathbf{u}}^{1}\left(\mathbf{z}_{\alpha}, \pm \frac{1}{2}\right)+\cdots
\end{aligned}
$$

${ }_{494}$ By identifying the terms in t - same powers of $\varepsilon$, Eq. A.26 gives:

$$
\begin{aligned}
{ }^{\prime}\left(\mathbf{x}_{o}, 0^{ \pm}\right) & =\hat{\mathbf{u}}^{0}\left(\mathbf{z}_{\alpha}, \pm \frac{1}{2}\right)=\overline{\mathbf{u}}^{0}\left(\mathbf{z}_{\alpha}, \pm \frac{1}{2}\right) \\
\left.\mathbf{u}^{1}\left(\mathbf{x}_{\alpha}, 0^{ \pm}\right) \pm{ }_{\llcorner}^{1} \mathbf{l}^{0}{ }^{\prime} \mathbf{x}_{\alpha}, 0^{ \pm}\right) & =\hat{\mathbf{u}}^{1}\left(\mathbf{z}_{\alpha}, \pm \frac{1}{2}\right)=\overline{\mathbf{u}}^{1}\left(\mathbf{z}_{\alpha}, \pm \frac{1}{2}\right)
\end{aligned}
$$

By identification ${ }_{\mathbf{r}}$ ces $_{\mathbf{c}}$, analogous results are obtained in terms of stresses [23, 25]:

$$
\sigma_{i 3}^{0}\left(\mathbf{x}_{\alpha}, 0^{ \pm}\right)=\hat{\sigma}_{i 3}^{0}\left(\mathbf{z}_{\alpha}, \pm \frac{1}{2}\right)=\bar{\sigma}_{i 3}^{0}\left(\mathbf{z}_{\alpha}, \pm \frac{1}{2}\right)
$$


497

$$
\sigma_{i 3}^{1}\left(\mathbf{x}_{\alpha}, 0^{ \pm}\right) \pm \frac{1}{2} \sigma_{i 3,3}^{0}\left(\mathbf{x}_{\alpha}, 0^{ \pm}\right)=\hat{\sigma}_{i 3}^{1}\left(\mathbf{z}_{\alpha}, \pm \frac{1}{2}\right)=\bar{\sigma}_{i 3}^{1}\left(\mathbf{z}_{\alpha}, \pm \frac{1}{2}\right)
$$

498

for $i=1,2,3$.

\section{A.5. Constitutive equations}

The constitutive laws in linear elasticity for the adherents an the nterphase are considered:

$$
\begin{aligned}
& \overline{\boldsymbol{\sigma}}^{\varepsilon}=\mathbb{A}_{ \pm}\left(\mathbf{e}\left(\overline{\mathbf{u}}^{\varepsilon}\right)\right) \\
& \hat{\boldsymbol{\sigma}}^{\varepsilon}=\mathbb{B}^{\varepsilon}\left(\mathbf{e}\left(\hat{\mathbf{u}}^{\varepsilon}\right)\right)
\end{aligned}
$$

where $\mathbb{A}_{ \pm}, \mathbb{B}^{\varepsilon}$ are the elasticity tensor of adher^nt a f of interphase, respectively.

Funding: This work has been supported $b_{y}$ the University of Ferrara via FAR grants 2020 and 2021.

\section{References}

[1] Sekiguchi, Y., Sato, C. ( $\therefore$ ^1) Experimental investigation of the effects of adhesive thickness on he, acture behavior of structural acrylic adhesive joints under varion o oadı., rates, Int. J. Adhes. Adhes., 105:102782.

[2] Miao, C., Ferna.. 'า, D., Heitzmann, M. T., Bailleres, H. (2019) GFRPto-timber h. d, l joints: Adhesive selection, Int. J. Adhes. Adhes., 94:2939. 
[3] Yamamoto, A., Yoshida, T., Tsubota, K., Takamizawa, T., Kurokawa, H., Miyazaki, M. (2006) Orthodontic bracket bonding: enamel bond strength vs time, Am. J. Orthod. Dentofacial Orth., 130(4):435-e?

[4] Benveniste, Y. and Miloh, T. (2001) Imperfect soft and stiff ..'orfa'ss in two-dimensional elasticity, Mech. Mat., 33 (6):309-323

[5] Hashin, Z. (2002) Thin interphase/imperfect interface: towsticity with application to coated fiber composites, J. Mech. Phys. Soı_.., 50(12):25092537.

[6] Frémond, M. (1987) Adhesion of solids, J. Mec'`. 1 1. Appl., 6(3):383407.

[7] Bonetti E., Bonfanti G., Lebon F., Rizzonı, '.. (2017) A model of imperfect interface with damage, Meccan `

[8] Raous, M., Cangemi, L., Cocu, M. (19؟y) A consistent model coupling adhesion,friction, and unilater ${ }^{-1}$ contact, Comput. Meth. Appl. Mech. Eng., 177(3-4):383-399.

[9] Del Piero, G., Raous, M. ( ( '10) A unified model for adhesive interfaces with damage, viscosity, u. $d$ friction, Eur. J.Mech.-A/Solid, 29(4):496507.

[10] Freddi, F., Frémor ㄱ. I.. (2006) Damage in domains and interfaces: a coupled predicti, theory, J. Mech. Mat. Struct., 1(7):1205-1233.

[11] Raffa, M.L., L' 's, F., Rizzoni R. (2016) On modelling brick/mortar 
interface via a St. Venant-Kirchhoff orthotropic soft interface. Part I: theory, Int. J. Masonry Res. Innov., 1 (2):142-164.

[12] Dumont, S., Lebon, F., Raffa, M. L., Rizzoni, R. (2017) Towards nc nlinear imperfect interface models including micro-cracks and smc : rour 々ness, Annal Solid Struct. Mech., 9(1-2):13-27.

[13] Raffa, M.L., Lebon, F., Rizzoni R. (2017) On modelli. y u, ıck/mortar interface via a St. Venant-Kirchhoff orthotropic soft inı, ,ace. Part II: in silico analysis, Int. J. Masonry Res. Innov., 2(4’ '259-273.

[14] Raffa, M. L., Lebon, F., Rizzoni, R. (2018) Der: atic $n$ of a model of imperfect interface with finite strains and dal re $\boldsymbol{c}_{3}$ symptotic techniques: an application to masonry structures, Mt anic $\lrcorner, 53(7): 1645-1660$.

[15] Maurel-Pantel, A., Lamberti, M., Raff M L., Suarez, C., Ascione, F., Lebon, F. (2020) Modelling of a GFI ' Jesive connection by an imperfect soft interface model with initial damage, Comp. Struct., 239:112034.

[16] Needleman, A. (1990) An anc' 'is of tensile decohesion along an interface, J. Mech. Phys. Soli', 3ð(`' '289-324.

[17] Costanzo, F. (1998) A cu.' 'inıum theory of cohesive zone models: deformation and constitutin e eq ıations, Int. J. Engng. Sci., 36(15):1763-1792.

[18] Alfano G., Sacco E ,2r J6) Combining interface damage and friction in a cohesive-zone, ^del, Int. J. Numer. Meth. Engng. 68 (5):542-582

[19] Chen, P., Chu. S., Peng, J. (2016) Interface behavior of a thin-film 
bonded to a graded layer coated elastic half-plane, Int. J. Mech. Sci. 115:489-500.

[20] Chen, P., Peng, J., Chen, Z., Peng, G. (2019) On the interfacial heh.vior of a piezoelectric actuator bonded to a homogeneous half plar _ .ith in arbitrarily varying graded coating, Eng. Fract. Mech. 220:100̉b-

[21] Guo, W., Chen, P., Yu, L., Peng, G., Zhao, Y., Gao, F. (־)‘u, Numerical analysis of the strength and interfacial behaviour of au...ively bonded joints with varying bondline thicknesses, Int. J. Adl s. Adhes. 98:102553.

[22] Chen, P., Chen, S., Liu, H., Peng, J., Gao, F. (2 ?0) : he interface behavior of multiple piezoelectric films attachin, $t_{c} a_{,}$,ite-thickness gradient substrate J. Appl. Mech.-Trans. ASME, ¿־(1):(^1003.

[23] Lebon, F., Rizzoni, R. (2010) Asymp tic „nalysis of a thin interface: The case involving similar rigidity, 'n'. ' ' Engng. Sci., 48:473-486

[24] Lebon, F., Rizzoni, R. (2011) A ymptotic behavior of a hard thin linear elastic interphase: An energy . mroach, Int. J. Solid Struct., 48:441-449

[25] Rizzoni, R., Dumont, S., L . ^n F., Sacco, S. (2014) Higher order model for soft and hard interfac. Jnt. J. Solid Struct., 51:4137-4148

[26] Dumont, S., Rizzoni, - Lebon, F., Sacco, E. (2018) Soft and hard interface models . $f_{c}$ " bo ıded elements, Comp. Part B: Engng., 153:480490.

[27] Furtsev, A., 1. 'Jy, E. (2020) Variational approach to modelling soft 
and stiff interfaces in the Kirchhoff-Love theory of plates, Int. J. Solid Struct., 202:562-574.

[28] Rudoy, E., Shcherbakov, V. (2020) First-order shape derivativ? cf the energy for elastic plates with rigid inclusions and interfacial cr - 'Ap'l. Math. Optim., 1-28.

[29] Baranova, S., Mogilevskaya, S. G., Nguyen, T. H., Schil!: 1gсı, D. (2020) Higher-order imperfect interface modelling via complex . .riables based asymptotic analysis, Int. J. Engng. Sci., 157:10339?

[30] Raffa, M.L., Lebon, F., Vairo, G. (2016) Norn al cnd tangential stiffnesses of rough surfaces in contact via an 'm, re', ' $\dot{i}$ interface model, Int. J. Solid Struct., 87:245-253.

[31] Raffa, M. L., Rizzoni, R., Lebon, F. (2rn1) , Model of Damage for Brittle and Ductile Adhesives in Glued 'su.' 'oints, Technologies, 9(1):19.

[32] Kachanov, M., (1994) Elastic ş'ids with many cracks and related prob-

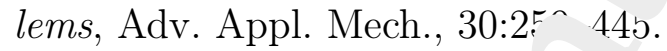

[33] Mauge C., Kachanov M '19'y4) Effective elastic properties of an anisotropic material with, , hitrarily oriented interacting cracks, J. Mech. Phys. Solid, 42:561-5`4.

[34] Tsukrov, I., Kach rov M. (2000) Effective moduli of an anisotropic

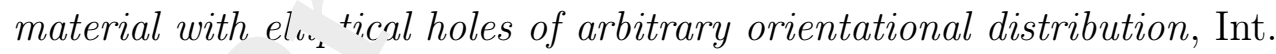
J. Solid St. •t., 37 (41):5919-5941. 
[35] Kachanov, M., Sevostianov, I. (2005) On quantitative characterization of microstructures and effective properties, Int. J. Solid Struct., 42(2):309336.

[36] Sevostianov, I., Kachanov, M. (2014) On some controversia' . 'ues in effective field approaches to the problem of the overall elastic $p$. ierties, Mech. Mat., 69:93-105.

[37] Kachanov, M., Sevostianov, I. (2018) Micromechanics o, ...uterials, with applications, (Vol. 249), Cham: Springer.

[38] Bruno, G., Kachanov, M., Sevostianov, I., Shyi m, ^. (2019) Micromechanical modelling of non-linear stress-st in ot. - vior of polycrystalline microcracked materials under tension, A七. Ma „erialia, 164:50-59.

[39] Andrieux, S., Bamberger, Y., Mari - J. J. (1986) Un modèle de matériau microfissuré pour les béton s .. 'cs roches, J. Méc. Théor. Appl, 5(3)47:1-513.

[40] Welemane, H., Goidescu, C. $\left(\wedge^{\wedge 10)}\right.$ Isotropic brittle damage and unilateral effect, Compte Renc' I Me 338(5):271-276.

[41] Sanchez-Palencia, E. $\left(1 \mathrm{\varkappa}^{\urcorner)}\right.$Non homogeneous materials and vibration theory, Lecture Notes in + 'yssics, Springer, Berlin, 127.

[42] Hubert, J. S., Pale 'ia E. S. (1992) Introduction aux méthodes asymptotiques et à l'h. naénéisation: application à la mécanique des milieux continus, $\mathrm{I} .{ }_{\mathrm{r}}^{\mathrm{r}} \mathrm{n}$. 
[43] Klarbring A. (1991) Derivation of the adhesively bonded joints by the asymptotic expansion method, Int. J. Engng. Sci., 29:493-512.

[44] Geymonat, G., Krasucki, F., Lenci, S. (1999) Mathematical annly.is of a bonded joint with a soft thin adhesive, Math. Mech. Solid, $1^{r}$.. `1-2‘ 5 .

[45] Schmidt, P. (2008) Modelling of adhesively bonded joints oy $n$ a. ymptotic method, Int. J. Engng. Sci., 46(12):1291-1324.

[46] Ciarlet P.G. (1988) Mathematical Elasticity. Volume I: ThreeDimensional Elasticity, North-Holland, Amsterdain.

[47] Serpilli, M., Lenci, S. (2008) Limit models in tru unalysis of three different layered elastic strips, Eur. J.Mech.-A, ``lia, 27(2):247-268.

[48] Serpilli, M., Lenci, S. (2016) An overvie' , af different asymptotic models for anisotropic three-layer plates with soj dhesive, Int. J. Solid Struct., 81:130-140.

[49] Goidescu, C., Welemane, H., Kcะ '’ D., Gruescu, C. (2013) Microcracks closure effects in initially orthot . pic materials, Eur. J.Mech.-A/Solid, $37: 172-184$.

[50] Goidescu, C., Welem - He, H., Pantalé, O., Karama, M., Kondo, D. (2015) Anisotropu "ilateral damage with initial orthotropy: A micromechanics-ba'd pproach, Int. J. Damage Mech., 24(3):313-337.

[51] Eshelby, J. ',. 196, Progress in solid mechanics, J. Mech. Phys. Solid, $9(1): 67-67$. 
[52] Welemane, H., Cormery, F. (2002) Some remarks on the damage unilateral effect modelling for microcracked materials, Int. J. Damage Mech., 11(1):65-86.

[53] Frémond, M., Nedjar, B. (1996) Damage, gradient of damage - v pr qciple of virtual power, Int. J. Solid Struct., 33(8):1083-1103.

[54] Chaboche, J. L. (1988) Continuum damage mechanics: ^aıı-General concepts, J. Appl. Mech., 55:59-64.

[55] Wolfram Research, Inc. Mathematica; Version 12.2; 'Nlfram Research, Inc.: Champaign, IL, USA, 2020.

[56] Murakami, S., Sekiguchi, Y., Sato, C., Yokı E., Furusawa, T. (2016) Strength of cylindrical butt joints bonded. $\lrcorner 2$ epoxy adhesives under combined static or high-rate loading, I+ J. Adhes. Adhes., 67:86-93. 